\title{
Analysis of Some Mixed Finite Element Methods Related to Reduced Integration*
}

\author{
By Claes Johnson and Juhani Pitkäranta
}

\begin{abstract}
We prove error estimates for the following two mixed finite element methods related to reduced integration: A method for Stokes' problem using rectangular elements with piecewise bilinear approximations for the velocities and piecewise constants for the pressure, and one method for a plate problem using bilinear approximations for transversal displacement and rotations and piecewise constants for the shear stress. The main idea of the proof in the case of Stokes' problem is to combine a weak Babuška-Brezzi type stability estimate for the pressure with a superapproximability property for the velocities. A similar technique is used in the case of the plate problem.
\end{abstract}

1. Introduction. In certain cases a direct application of the finite element method gives very inaccurate results. This happens, e.g., for displacement type finite element methods for thin plates constructed starting from a three-dimensional model of the plate. In this case the resulting discrete models will be much too stiff and hence the numerical results will be very poor. We find a similar phenomenon if we try to solve Stokes' equations approximately using piecewise bilinear trial functions satisfying the divergence zero condition exactly. The reason for failure in both cases is that in the discrete model some of the conditions are emphasized too much at the expense of other conditions, so that the model becomes "unbalanced" or "too stiff". In the case of Stokes' problem too much effort is spent on satisfying the divergence zero condition, and the approximability is seriously affected. For the plate problem too much emphasis is put on a compatibility condition between displacements and rotations.

In order to relax such conditions to obtain a "balanced" discrete model, the technique of selective reduced integration (see, e.g., [11], [14]) has been used widely in practice, often with considerable success. In the Stokes problem with bilinear trial functions, the relaxation is achieved by requiring only the mean value over each element (i.e., the value at the midpoint of each element) of the divergence to be zero. In the case of a plate problem using bilinear trial functions for displacements and rotations, the compatibility condition is relaxed and is required to hold only at the midpoint of each element. In both cases the so modified methods perform surprisingly well (however, these methods are somewhat "delicate" in the sense that extra smoothness of the exact solution is required; cf. below).

Received November 11, 1980.

1980 Mathematics Subject Classification. Primary 65N15, 65N30.

*This work was carried out while Juhani Pitkäranta was visiting Chalmers University of Technology during October 15-December 15, 1979. 
Methods of this type can be viewed as obtained by starting from a penalty formulation with a penalty term for the condition to be relaxed and then using a low-order integration formula for this term to achieve the relaxation. This is the motivation for the term selective reduced integration. Alternatively, these methods can be viewed (cf. [11]) as certain mixed finite element methods. In fact, this point of view seems to be the more general one and is also the one adopted below in the analysis.

The purpose of this note is to prove some error estimates for the two mixed methods related to reduced integration mentioned above. The only previous result in this direction, to our knowledge, was given in [15], where convergence (with no error estimates) for the velocities was demonstrated for a finite-difference analogue of the mixed method for Stokes' problem.

The analysis follows the general lines of Babuška [1] and Brezzi [4] but contains some nonstandard features. As usual when analyzing a mixed method, the difficulty is to verify some type of Babuška-Brezzi stability condition in order to get control of the "auxiliary variable" (the pressure in the case of Stokes' problem). Here we can only control this variable in a weak mesh-dependent seminorm, and we compensate for this weak estimate by using a "superapproximation" property for the main variable (the velocities in Stokes' problem). In the case of Stokes' problem, we obtain optimal rates of convergence in $L_{2}$ and $H^{1}$ for the velocities, i.e., $\theta\left(h^{2}\right)$ and $\theta(h)$ where $h$ is the mesh length, requiring relatively little extra smoothness. For the pressures computed in the natural way, we do not obtain any rate of convergence in $L_{2}$. However, we prove that a simple local averaging gives pressures with $L_{2}$-error of order $\theta(h)$. For the plate problem, we obtain $\Theta(h)$ convergence in $H^{1}$ for displacements and rotations and $\theta\left(h^{3 / 2}\right)$ convergence in $L_{2}$ for the displacements under considerable extra smoothness assumptions.

For simplicity we consider two model problems. The ideas used in the analysis can probably be used also to analyze some other mixed methods related to reduced integration such as, e.g., the analogous method for Stokes' problem using biquadratic velocities and bilinear pressures, cf. [11].

An outline of the paper is as follows: Section 2 contains some preliminaries, in Section 3 we treat Stokes' problem and in Section 4 the plate problem.

2. Preliminaries. Let us start by introducing some notation. Let $x_{0}$ and $y_{0}$ be positive numbers, and let $\Omega$ be the rectangle $\left\{(x, y) \in \mathbf{R}^{2}: 0<x<x_{0}, 0<y<y_{0}\right\}$. We introduce the usual Sobolev spaces $W^{k, p}(\Omega), k \geqslant 0,1 \leqslant p<\infty$, with norms

$$
\|v\|_{k, p}=\left(\sum_{l=0}^{k}|v|_{l, p}^{p}\right)^{1 / p}
$$

where $\mid \cdot l_{l, p}$ denotes the seminorms

$$
|v|_{l, p}=\left(\sum_{i+j=l} \int_{\Omega}\left|\frac{\partial^{i+j} v}{\partial x^{i} \partial y^{j}}\right|^{p} d x d y\right)^{1 / p} .
$$

For $p=2$ we set $H^{k}(\Omega)=W^{k, 2}(\Omega),|\cdot|_{k}=|\cdot|_{k, 2}$ and $\|\cdot\|_{k}=\|\cdot\|_{k, 2}$. The same notation will be used for the corresponding (semi)norms in $\left[W^{k, p}(\Omega)\right]^{2}$. The scalar products in $L_{2}(\Omega)$ or $\left[L_{2}(\Omega)\right]^{2}$ will be denoted by $(\cdot, \cdot)$. As usual $H_{0}^{k}(\Omega), k \geqslant 1$, 
denotes the completion of $C_{0}^{\infty}(\Omega)$ in the norm $\|\cdot\|_{k}$ and $H^{-k}(\Omega)$ denotes the dual of $H_{0}^{k}(\Omega)$ with norm $\|\cdot\|_{-k}$.

Finally, by $C$ or $C_{j}$ we denote positive constants, possibly different at different occurrences, which may depend on $\Omega$ but not on any other parameter to be introduced unless indicated explicitly.

Let us now introduce some finite element spaces to be used below. For simplicity we shall consider partitions of the rectangle $\Omega$ into rectangular elements with uniform partitions in the $x$ - and $y$-direction. Let $\mathcal{C}_{h}^{0}$ be the uniform partition obtained by using rectangles of size $h_{1} \times h_{2}$, i.e.,

$$
\begin{gathered}
\bigodot_{h}^{0}=\left\{K_{i j}: i=1, \ldots, n, j=1, \ldots, m\right\}, \\
K_{i j}=\left\{(x, y) \in \mathbf{R}^{2}:(i-1) h_{1}<x<i h_{1},(j-1) h_{2}<y<j h_{2}\right\},
\end{gathered}
$$

where $n=x_{0} / h_{1}$ and $m=y_{0} / h_{2}$ are integers. We shall assume that $h_{1}$ and $h_{2}$ depend on the mesh parameter $h \equiv h_{1} \in(0,1)$ in such a way that $h_{1} / h_{2}$ is bounded by positive constants from below and above independent of $h$. The finite element spaces to be introduced will be associated with the partition $\bigodot_{h}$ obtained by dividing each $K_{i j} \in \mathcal{C}_{h}^{0}$ into four equal subrectangles:

$$
\begin{gathered}
\mathcal{C}_{h}=\left\{\Delta_{i j}: i=1, \ldots, 2 m, j=1, \ldots, 2 n\right\}, \\
\Delta_{i j}=\left\{(x, y) \in \mathbf{R}^{2}:(i-1) h_{1} / 2<x<i h_{1} / 2,(j-1) h_{2} / 2<y<j h_{2} / 2\right\} .
\end{gathered}
$$

Let us now define

$$
\begin{aligned}
& S_{h}=\left\{v \in H_{0}^{1}(\Omega):\left.v\right|_{\Delta_{i j}} \text { is bilinear } \forall \Delta_{i j} \in \mathcal{C}_{h}\right\}, \\
& T_{h}=\left\{\mu \in L_{2}(\Omega):\left.\mu\right|_{\Delta_{i j}} \text { is constant } \forall \Delta_{i j} \in \mathcal{C}_{h}\right\} .
\end{aligned}
$$

These spaces will be the building blocks in the finite element methods below.

We will need an a priori estimate for the solution of the following biharmonic problem:

$$
\left\{\begin{array}{l}
\Delta^{2} u=f, \\
u \in H_{0}^{2}(\Omega),
\end{array}\right.
$$

where $f \in H^{-2}(\Omega)$. We have (see [8], [10])

Proposition 2.1. If $u$ is the solution of (2.1), then

$$
\|u\|_{k+4} \leqslant C\|f\|_{k}, \quad-2 \leqslant k \leqslant 0 .
$$

\section{A Mixed Method for Stokes' Problem.}

3.1. Formulation of the Problem. Let us recall Stokes' equations for an incompressible viscous fluid with viscosity equal to one:

$$
\begin{cases}-\Delta u+\nabla \lambda=f & \text { in } \Omega \\ \operatorname{div} u=0 & \text { in } \Omega \\ u=0 & \text { on } \partial \Omega \\ \int_{\Omega} \lambda d x=0 . & \end{cases}
$$


Here $u=\left(u_{1}, u_{2}\right)$ is the velocity and $\lambda$ the pressure of the fluid. For simplicity we consider Dirichlet boundary conditions, and we also normalize the pressure to have zero mean value. In variational form $(3.1)$ reads: Find $(u, \lambda) \in\left[H_{0}^{1}(\Omega)\right]^{2} \times L_{2}(\Omega)$ such that

$$
\begin{cases}(\nabla u, \nabla v)-(\lambda, \operatorname{div} v)=(f, v) & \forall v \in\left[H_{0}^{1}(\Omega)\right]^{2}, \\ (\mu, \operatorname{div} u)=0 & \forall \mu \in L_{2}(\Omega), \\ \int_{\Omega} \lambda d x=0 . & \end{cases}
$$

Let now $V_{h}=\left[S_{h}\right]^{2}, Q_{h}=T_{h}$, and let us formulate the following finite element method for Stokes' problem: Find $\left(u_{h}, \lambda_{h}\right) \in V_{h} \times Q_{h}$ such that

$$
\begin{cases}\left(\nabla u_{h}, \nabla v\right)-\left(\lambda_{h}, \operatorname{div} v\right)=(f, v) & \forall v \in V_{h}, \\ \varepsilon\left(\lambda_{h}, \mu\right)+\left(\mu, \operatorname{div} u_{h}\right)=0 & \forall \mu \in Q_{h},\end{cases}
$$

where $\varepsilon$ is a small positive parameter to be specified below. We note that (3.3) may be considered to be a discrete analogue of the perturbed Stokes problem:

$$
\begin{cases}-\Delta u+\nabla \lambda=f & \text { in } \Omega \\ \varepsilon \lambda+\operatorname{div} u=0 & \text { in } \Omega \\ u=0 & \text { on } \partial \Omega\end{cases}
$$

corresponding to an almost incompressible fluid (cf. [3]).

To see the connection with reduced integration in (3.3) we note that, solving for $\lambda_{h}$ in (3.3b), which can be done locally on each element, and eliminating $\lambda_{h}$ in (3.3a), we obtain an equation for $u_{h} \in V_{h}$ which can be formulated as follows:

$$
\left(\nabla u_{h}, \nabla v\right)+\frac{1}{\varepsilon}\left(\operatorname{div} u_{h}, \operatorname{div} v\right)_{*}=(f, v) \quad \forall v \in V_{h},
$$

where $(\cdot, \cdot)_{*}$ indicates that the scalar product is evaluated using the simple quadrature rule (one-point Gaussian quadrature):

$$
\int_{\Delta} v d x=v(M) h_{1} h_{2} / 4, \quad M \text { midpoint of } \Delta \in \mathcal{C}_{h} .
$$

The solution $u_{h} \in V_{h}$ of (3.4) can equivalently be characterized as the solution of the minimization problem

$$
\operatorname{Min}_{v \in V_{h}}\left\{\frac{1}{2}(\nabla v, \nabla v)+\frac{1}{2 \varepsilon}(\operatorname{div} v, \operatorname{div} v)_{*}-(f, v)\right\} .
$$

Now, this problem can also be viewed as being obtained by using selective reduced integration in the problem

$$
\operatorname{Min}_{v \in V_{h}}\left\{\frac{1}{2}(\nabla v, \nabla v)+\frac{1}{2 \varepsilon}(\operatorname{div} v, \operatorname{div} v)-(f, v)\right\}
$$

which is a standard penalty method for Stokes' problem. Comparing (3.5) and (3.6), let us remark that in order to get reasonable results using (3.6) one has to tie $\varepsilon$ to the mesh parameter $h$. If $\varepsilon$ is chosen too small, the penalty becomes too dominant and the results will be useless. However, one has to choose $\varepsilon$ reasonably small to enforce the divergence zero condition approximately. Even with optimal choice of $\varepsilon$ the 
method (3.6) will give only suboptimal rates of convergence $\left(\theta(\sqrt{h})\right.$ in $H^{1}$-norm). On the other hand, we shall prove below that if only $\varepsilon$ is sufficiently small $\left(\varepsilon \leqslant C h^{2}\right)$, then the relaxed method (3.5) will be optimal in $H^{1}$ and $L_{2}(\Omega)$ for the velocities $\left(\theta(h)\right.$ and $\theta\left(h^{2}\right)$, respectively). In particular, this method does not become illconditioned as $\varepsilon$ gets small as is the case with (3.6). In practice a lower limit for $\varepsilon h$ is set by the available machine precision. For related ideas in connection with more conventional mixed methods see [3].

The existence of a unique solution of (3.3) for $\varepsilon>0$ follows from the stability estimate

$$
\left\|u_{h}\right\|_{1}+\sqrt{\varepsilon}\left\|\lambda_{h}\right\|_{0} \leqslant C\|f\|_{-1},
$$

obtained by taking $v=u_{h}$ and $\mu=\lambda_{h}$ in (3.3). If $\varepsilon=0$, however, then $\lambda_{h}$ is not uniquely determined but has two undetermined degrees of freedom (cf. Remark 3.1 below).

3.2. A Basic Error Estimate. Let us now analyze the finite element method (3.3) considered as a discrete analogue of the unperturbed Stokes problem (3.2). We shall then need the following a priori estimate for the solution of (3.2):

$$
\|u\|_{k+2}+\|\lambda\|_{k+1} \leqslant C\|f\|_{k}, \quad k=0,1 .
$$

This estimate follows from Proposition 2.1 using the stream-function-vorticity formulation of Stokes' equations.

As a first step let us introduce a special orthogonal basis for the space $Q_{h}$ of piecewise constants, which will be of crucial importance in the subsequent analysis. The basis consists of the functions $\xi_{i j k} \in Q_{h}, i=1, \ldots, n, j=1, \ldots, m, k=1, \ldots, 4$, defined as follows: The support of each $\xi_{i j k}, k=1, \ldots, 4$, is contained in $K_{i j} \in \bigodot_{h}^{0}$, and on $K_{i j}$ the functions $\xi_{i j k}, k=1, \ldots, 4$, take the values \pm 1 on the four subrectangles of $K_{i j}$ according to the following pattern:
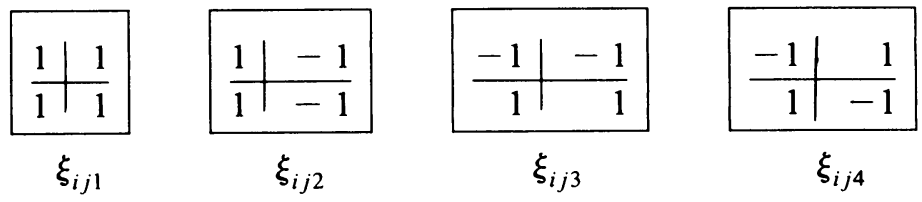

FIGURE 1

The values on $K_{i j}$ of the basis functions $\xi_{i j k}, k=1, \ldots, 4$

Any $\mu \in Q_{h}$ has the unique representation

$$
\mu=\sum_{i, j, k} \alpha_{i j k} \xi_{i j k}, \quad \alpha_{i j k} \in \mathbf{R}
$$

Here and below we sum $i, j$ and $k$ from 1 to $n, m$ and 4 , respectively (in Section 4 below, $k$ will run from 1 to 8 ).

Next, let us introduce the following subspaces of $Q_{h}$ :

$$
\begin{aligned}
& N_{h}=\left\{\mu \in Q_{h}:(\mu, \operatorname{div} v)=0 \forall v \in V_{h}\right\}, \\
& N_{h}^{\perp}=\left\{\lambda \in Q_{h}:(\lambda, \mu)=0 \forall \mu \in N_{h}\right\} .
\end{aligned}
$$


It is easy to see that $N_{h}$ is a two-dimensional space (cf. proof of Theorem 3.1 below) with the orthogonal basis functions $\varphi_{1}$ and $\varphi_{2}$ given by

$$
\begin{array}{ll}
\varphi_{1}(x)=1, & x \in \Omega, \\
\varphi_{2}(x)=(-1)^{i+j}, & x \in \Delta_{i j} \in \mathcal{C}_{h} .
\end{array}
$$

We can then characterize the space $N_{h}^{\perp}$ as follows:

$$
N_{h}^{\perp}=\left\{\sum_{i, j, k} \alpha_{i j k} \xi_{i j k}: \sum_{i, j} \alpha_{i j 1}=0, \sum_{i, j} \alpha_{i j 4}=0\right\} .
$$

The presence of the "checker-board" function $\varphi_{2}$ in the nullspace $N_{h}$ was noted in [13].

Remark 3.1. Clearly there exists a unique pair $\left(\bar{u}_{h}, \bar{\lambda}_{h}\right) \in V_{h} \times N_{h}^{\perp}$ that satisfies (3.3) with $\varepsilon=0$. Denoting by $\left(u_{h}^{\varepsilon}, \lambda_{h}^{\varepsilon}\right)$ the solution of (3.3) with $\varepsilon>0$, we have by (3.3b) that $\lambda_{h}^{\varepsilon} \in N_{h}^{\perp}$ and furthermore $\left(u_{h}^{\varepsilon}, \lambda_{h}^{\varepsilon}\right) \rightarrow\left(\bar{u}_{h}, \bar{\lambda}_{h}\right)$ as $\varepsilon \rightarrow 0$. Thus, the problem (3.3) with $\varepsilon$ small and positive, which after elimination of the pressure corresponds to a positive definite linear system (cf. (3.4)), can be viewed as a computationally convenient form of the problem (3.3) with $\varepsilon=0$ and the requirement $\lambda_{h} \in N_{h}^{\perp}$.

We will supply $Q_{h}$ with the mesh-dependent seminorm $|\cdot|_{h}$ defined by

$$
|\mu|_{h}^{2}=\sum_{k=1}^{3}\left\|\mu_{k}\right\|_{0}^{2}+h^{2} \sigma\left(\mu_{4}\right)^{2}, \quad \mu=\sum_{i, j, k} \alpha_{i j k} \xi_{i j k},
$$

where

$$
\mu_{k}=\sum_{i, j} \alpha_{i j k} \xi_{i j k}, \quad k=1, \ldots, 4
$$

and

$$
\sigma\left(\mu_{4}\right)^{2}=\sum_{i=1}^{n-1} \sum_{j}\left(\alpha_{i j 4}-\alpha_{i+1, j 4}\right)^{2}+\sum_{i} \sum_{j=1}^{m-1}\left(\alpha_{i j 4}-\alpha_{i, j+1,4}\right)^{2} .
$$

Clearly, $|\cdot|_{h}$ is a norm on $N_{h}^{\perp}$, and, comparing this norm with the $L_{2}$-norm $\|\cdot\|_{0}$, we easily see that (cf. Lemma 3.3 below) for $\mu \in N_{h}^{\perp}$

$$
\begin{gathered}
C_{1} h\|\mu\|_{0} \leqslant|\mu|_{h} \leqslant C_{2}\|\mu\|_{0}, \\
|\mu|_{h}=\|\mu\|_{0} \text { if } \mu_{4}=0 .
\end{gathered}
$$

The proof of the basic error estimate for the method (3.3) will be based on the following Babuška-Brezzi (cf. [1], [4]) type stability estimate:

LEMMA 3.1. There is a constant $C$ such that

$$
\sup _{v \in V_{h}} \frac{(\mu, \operatorname{div} v)}{\|v\|_{1}} \geqslant C|\mu|_{h}
$$

for all $\mu \in Q_{h}$ such that $(\mu, 1)=0$.

In the proof of this result we shall use the following easy-to-prove (cf. [6], [7], [8]) analogue of Lemma 3.1 obtained by replacing $Q_{h}$ by $Q_{h}^{1}$, where $Q_{h}^{1}$ consists of the functions in $Q_{h}$ which are constant on each $K_{i j} \in \mathcal{C}_{h}^{0}$, i.e., $Q_{h}^{1}=\left\{\mu_{1}: \mu \in Q_{h}\right\}$. 
LEMMA 3.2. There is a constant $C$ such that

$$
\sup _{v \in V_{h}} \frac{(\mu, \operatorname{div} v)}{\|v\|_{1}} \geqslant C\|\mu\|_{0},
$$

for all $\mu \in Q_{h}^{1}$ satisfying $(\mu, 1)=0$.

Proof. Given $\mu \in Q_{h}^{1}$ with $(\mu, 1)=0$, there exists (cf. [8]) $z \in\left[H_{0}^{1}(\Omega)\right]^{2}$ such that

$$
\operatorname{div} z=\mu \text { in } \Omega, \quad\|z\|_{1} \leqslant C\|\mu\|_{0} .
$$

Let us now define $z_{h} \in V_{h}$ by requiring

$$
\begin{aligned}
& z_{h}(P)=w_{h}(P) \quad \text { if } P \text { is a corner or the midpoint of } K_{i j} \in \mathcal{C}_{h}^{0}, \\
& \int_{S} z_{h} d s=\int_{S} z d s \quad \text { if } S \text { is a side of } K_{i j} \in \mathcal{C}_{h}^{0},
\end{aligned}
$$

where $w_{h} \in V_{h}$ satisfies $\left(\nabla z-\nabla w_{h}, \nabla v\right)=0 \forall v \in V_{h}$. One can then verify (cf. [6], [8]) that $z_{h}$ is well defined and that

$$
\left\|z_{h}\right\|_{1} \leqslant C\|z\|_{1}, \quad\left(\operatorname{div} z_{h}, \mu\right)=(\operatorname{div} z, \mu) \quad \forall \mu \in Q_{h}^{1} .
$$

Thus we have

$$
\frac{\left(\mu, \operatorname{div} z_{h}\right)}{\left\|z_{h}\right\|_{1}} \geqslant C \frac{(\mu, \operatorname{div} z)}{\|z\|_{1}} \geqslant C\|\mu\|_{0},
$$

which proves the lemma.

Proof of Lemma 3.1. Let $\mu=\Sigma_{i j k} \alpha_{i j k} \xi_{i j k}=\Sigma_{k} \mu_{k}$ be given with $(\mu, 1)=0$. We first define two functions $z=\left(z_{1}, z_{2}\right) \in V_{h}$ and $w=\left(w_{1}, w_{2}\right) \in V_{h}$ as follows:

$$
\left\{\begin{array}{l}
z_{1}(P)=h \alpha_{i j 2} \\
z_{2}(P)=h \alpha_{i j 3}
\end{array} \text { if } P \text { is the midpoint of } K_{i j} \in \mathcal{C}_{h}^{0},\right.
$$

$$
w_{2}(P)=h\left(\alpha_{i+1, j 4}-\alpha_{i j 4}\right) \text { if } P \text { is the midpoint of the common side }
$$

$$
\text { of } K_{i j} \text { and } K_{i+1, j} \in \bigodot_{h}^{0},
$$

$$
w_{1}(P)=h\left(\alpha_{i, j+1,4}-\alpha_{i j 4}\right) \text { if } P \text { is the midpoint of the common side }
$$

$$
\text { of } K_{i j} \text { and } K_{i, j+1} \in \mathcal{C}_{h}^{0} \text {, }
$$

(iv) the remaining degrees of freedom of $z$ and $w$ are equal to zero.

It is straightforward to verify from the above definitions that the following inequalities hold:

$$
\begin{gathered}
\|z\|_{1} \leqslant C\left(\left\|\mu_{2}\right\|_{0}^{2}+\left\|\mu_{3}\right\|_{0}^{2}\right)^{1 / 2}, \quad\|w\|_{1} \leqslant C h \sigma\left(\mu_{4}\right), \\
(\mu, \operatorname{div} z)=\left(\mu_{2}+\mu_{3}, \operatorname{div} z\right) \geqslant C\left(\left\|\mu_{2}\right\|_{0}^{2}+\left\|\mu_{3}\right\|_{0}^{2}\right),
\end{gathered}
$$

and

$$
\begin{aligned}
(\mu, \operatorname{div} w) & =\left(\sum_{k=2}^{4} \mu_{k}, \operatorname{div} w\right) \geqslant C h^{2} \sigma\left(\mu_{4}\right)^{2}+\left(\mu_{2}+\mu_{3}, \operatorname{div} w\right) \\
& \geqslant C_{1} h^{2} \sigma\left(\mu_{4}\right)^{2}-C_{2}\left(\left\|\mu_{2}\right\|_{0}^{2}+\left\|\mu_{3}\right\|_{0}^{2}\right)
\end{aligned}
$$


To proceed, we need a third function $g=\left(g_{1}, g_{2}\right) \in V_{h}$ satisfying

$$
\|g\|_{1} \leqslant C\left\|\mu_{1}\right\|_{0}, \quad\left(\mu_{1}, \operatorname{div} g\right) \geqslant C\left\|\mu_{1}\right\|_{0}^{2} .
$$

Since $\left(\mu_{1}, 1\right)=(\mu, 1)=0$, the existence of $g$ follows from Lemma 3.2.

Now, let $v=z+\delta w+\delta^{2} g$, where $\delta \in(0,1]$ will be chosen below. Then we have

$$
\|v\|_{1} \leqslant C|\mu|_{h}
$$

and

$$
\begin{aligned}
(\mu, \operatorname{div} v) \geqslant & C \delta^{2}\left\|\mu_{1}\right\|_{0}^{2}+\left(C-C_{1} \delta\right)\left(\left\|\mu_{2}\right\|_{0}^{2}+\left\|\mu_{3}\right\|_{0}^{2}\right) \\
& +C \delta h^{2} \sigma\left(\mu_{4}\right)^{2}+\delta^{2}\left(\mu_{4}, \operatorname{div} g\right) .
\end{aligned}
$$

To finally estimate $\left(\mu_{4}, \operatorname{div} g\right)$, let $g_{k i j}=g_{k}\left(i h_{1} / 2, j h_{2} / 2\right), i=0, \ldots, 2 n, j=$ $0, \ldots, 2 m$. By a straightforward computation we find that

$$
\begin{aligned}
\left(\mu_{4}, \operatorname{div} g\right)= & \frac{1}{2} h_{1} \sum_{i=1}^{n-1} \sum_{j=1}^{m}\left(\alpha_{i j 4}-\alpha_{i+1, j 4}\right) \\
& \times\left(g_{2,2 i, 2 j-2}-2 g_{2,2 i, 2 j-1}+g_{2,2 i, 2 j}\right) \\
& +\frac{1}{2} h_{2} \sum_{i=1}^{n} \sum_{j=1}^{m-1}\left(\alpha_{i, j+1,4}-\alpha_{i j 4}\right) \\
& \times\left(g_{1,2 i-2,2 j}-2 g_{1,2 i-1,2 j}+g_{1,2 i, 2 j}\right),
\end{aligned}
$$

and therefore

$$
\begin{aligned}
\left(\mu_{4}, \operatorname{div} g\right) & \leqslant C h \sigma\left(\mu_{4}\right)\left\{\sum_{i=0}^{2 n-1} \sum_{j=0}^{2 m-1} \sum_{k=1}^{2}\left[\left(g_{k i j}-g_{k, i+1, j}\right)^{2}+\left(g_{k i j}-g_{k i, j+1}\right)^{2}\right]\right\}^{1 / 2} \\
& \leqslant C_{1} h \sigma\left(\mu_{4}\right)|g|_{1} \leqslant C_{2} h \sigma\left(\mu_{4}\right)\left\|\mu_{1}\right\|_{0} .
\end{aligned}
$$

Thus,

$$
(\mu, \operatorname{div} v) \geqslant\left(C-C_{1} \delta\right)\left[\delta^{2}\left\|\mu_{1}\right\|_{0}^{2}+\left\|\mu_{2}\right\|_{0}^{2}+\left\|\mu_{3}\right\|_{0}^{2}+\delta h^{2} \sigma\left(\mu_{4}\right)^{2}\right] .
$$

Choosing now $\delta=\min \left\{1, C / 2 C_{1}\right\}$, we see that $(\mu, \operatorname{div} v) \geqslant C|\mu|_{h}^{2}$, which together with (3.10) proves the lemma.

Remark 3.2. From the proof of Lemma 3.1 we see that if $\mu \in Q_{h}$ and $v \in V_{h}$, then $(\mu, \operatorname{div} v) \leqslant C|\mu|_{h}|v|_{1}$. Therefore we can actually state that, for $\mu \in Q_{h}$ with $(\mu, 1)=0$,

$$
C_{1}|\mu|_{h} \geqslant \sup _{v \in V_{h}} \frac{(\mu, \operatorname{div} v)}{\|v\|_{1}} \geqslant C_{2}|\mu|_{h} .
$$

As a final preparation for the proof of the basic error estimate we note the following discrete Sobolev imbedding result:

Lemma 3.3. For $1 \leqslant q<\infty$, there is a positive constant $C(q)$ such that if $\Sigma_{i, j} \alpha_{i j}=0$, then

$$
\left\{\sum_{i=1}^{n-1} \sum_{j}\left(\alpha_{i j}-\alpha_{i+1, j}\right)^{2}+\sum_{i} \sum_{j=1}^{m-1}\left(\alpha_{i j}-\alpha_{i, j+1}\right)^{2}\right\}^{1 / 2} \geqslant C(q) h^{2 / q}\left\{\sum_{i, j}\left|\alpha_{i j}\right|^{q}\right\}^{1 / q}
$$


Proof. Let $C_{h}^{1}$ be another rectangular partitioning of $\Omega$, the interior nodes of which are located at the midpoints of each $K_{i j} \in \mathcal{C}_{h}^{0}$, and let $v$ be the continuous piecewise bilinear function on $\bigodot_{h}^{1}$ defined by $v(P)=\alpha_{i j}$ if $P$ is a node of $\bigodot_{h}^{1}$ contained in $\overline{K_{i j}}$, $K_{i j} \in \mathcal{C}_{h}^{0}$. Then it is easy to see that

$$
\int_{\Omega} v d x=h_{1} h_{2} \sum_{i, j} \alpha_{i j}=0,
$$

and therefore, by Poincaré's and Sobolev's inequalities,

$$
|v|_{1} \geqslant C\|v\|_{1} \geqslant C(q)\|v\|_{0, q}, \quad q<\infty .
$$

Using the obvious inequalities

$$
\begin{gathered}
\|v\|_{0, q} \geqslant C h^{2 / q}\left\{\sum_{i, j}\left|\alpha_{i j}\right|^{q}\right\}^{1 / q}, \\
|v|_{1} \leqslant C\left\{\sum_{i=1}^{n-1} \sum_{j}\left(\alpha_{i j}-\alpha_{i+1, j}\right)^{2}+\sum_{i} \sum_{j=1}^{m-1}\left(\alpha_{i j}-\alpha_{i, j+1}\right)^{2}\right\}^{1 / 2},
\end{gathered}
$$

the desired estimate follows.

We can now state and prove the basic error estimate for the method (3.3).

THEOREM 3.1. Assume that the solution of (3.2) satisfies $(u, \lambda) \in\left[W^{3, p}(\Omega)\right]^{2} \times$ $H^{1}(\Omega)$ for some $p>1$. Then if $\left(u_{h}, \lambda_{h}\right)$ is the solution of (3.3) with $0<\varepsilon \leqslant C h^{2}$ and $\tilde{\lambda} \in N_{h}^{\perp}$ is the orthogonal projection of $\lambda$ onto $N_{h}^{\perp}$, we have

$$
\left|u-u_{h}\right|_{1}+\left|\lambda_{h}-\tilde{\lambda}\right|_{h} \leqslant C(p) h\left(|u|_{2}+|u|_{3, p}+\|\lambda\|_{1}\right)
$$

Proof. Let $\tilde{u} \in V_{h}$ be the usual interpolant of $u$, and let $\tilde{\lambda}$ be the orthogonal projection (in $L_{2}(\Omega)$ ) of $\lambda$ onto $N_{h}^{\perp}$. From (3.3) and (3.2) we have the following identity:

$$
\begin{aligned}
& \mathscr{B}\left(u_{h}-\tilde{u}, \lambda_{h}-\tilde{\lambda} ; v, \mu\right) \\
& \quad=\mathscr{B}(u-\tilde{u}, \lambda-\tilde{\lambda} ; v, \mu)-\varepsilon(\lambda, \mu) \quad \forall(v, \mu) \in V_{h} \times Q_{h},
\end{aligned}
$$

where

$$
\Re(u, \lambda ; v, \mu)=(\nabla u, \nabla v)-(\lambda, \operatorname{div} v)+(\mu, \operatorname{div} u)+\varepsilon(\lambda, \mu)
$$

Since $\left(\lambda_{h}-\tilde{\lambda}, 1\right)=0$, there exists, by Lemma 3.1, $z \in V_{h}$ satisfying

$$
\|z\|_{1} \leqslant C\left|\lambda_{h}-\tilde{\lambda}\right|_{h}, \quad-\left(\lambda_{h}-\tilde{\lambda}, \operatorname{div} z\right) \geqslant\left|\lambda_{h}-\tilde{\lambda}\right|_{h}^{2} .
$$

Let us now define

$$
\mathscr{H}=\left\{\left|u_{h}-\tilde{u}\right|_{1}^{2}+\left|\lambda_{h}-\tilde{\lambda}\right|_{h}^{2}+\varepsilon\left\|\lambda_{h}-\tilde{\lambda}\right\|_{0}^{2}\right\}^{1 / 2},
$$

and let $v=u_{h}-\tilde{u}+\delta z$ and $\mu=\lambda_{h}-\tilde{\lambda}$, where $\delta \in(0,1]$ will be chosen below. Then we have

$$
\|v\|_{1}+|\mu|_{h}+\sqrt{\varepsilon}\|\mu\|_{0} \leqslant C \mathcal{H}
$$


and

$$
\begin{aligned}
\mathscr{B}\left(u_{h}-\tilde{u}, \lambda_{h}\right. & -\tilde{\lambda} ; v, \mu) \\
& =\left|u_{h}-\tilde{u}\right|_{1}^{2}+\delta\left|\lambda_{h}-\tilde{\lambda}\right|_{h}^{2}+\varepsilon\left\|\lambda_{h}-\tilde{\lambda}\right\|_{0}^{2}+\delta\left(\nabla\left(u_{h}-\tilde{u}\right), \nabla z\right) \\
& \geqslant\left(C-C_{1} \delta\right)\left|u_{h}-\tilde{u}\right|_{1}^{2}+\frac{1}{2} \delta\left|\lambda_{h}-\tilde{\lambda}\right|_{h}^{2}+\varepsilon\left\|\lambda_{h}-\tilde{\lambda}\right\|_{0}^{2} .
\end{aligned}
$$

Choosing now $\delta=\min \left\{1,1 / 2 C_{1}\right\}$, we find that

$$
\mathscr{B}\left(u_{h}-\tilde{u}, \lambda_{h}-\tilde{\lambda} ; v, \mu\right) \geqslant C \mathcal{K}^{2} .
$$

Next, let us estimate the right-hand side of (3.12). First, using (3.14) and the inequality $\varepsilon \leqslant C h^{2}$ together with (3.8), we see that

$$
\begin{aligned}
& |\mathfrak{B}(u-\tilde{u}, \lambda-\tilde{\lambda} ; v, \mu)-\varepsilon(\lambda, \mu)| \\
& \quad \leq C \mathcal{H}|u-\tilde{u}|_{1}+|(\lambda-\tilde{\lambda}, \operatorname{div} v)|+|(\mu, \operatorname{div}(u-\tilde{u}))|+C h \mathcal{H}\|\lambda\|_{0} .
\end{aligned}
$$

For the first term on the right-hand side we have by the well-known interpolation theory [5]

$$
|u-\tilde{u}|_{1} \leqslant C h|u|_{2} .
$$

To estimate the second term, we note that if $\pi_{h} \lambda$ is the orthogonal projection of $\lambda$ onto $Q_{h}$, then

$$
(\tilde{\lambda}, \operatorname{div} v)=\left(\pi_{h} \lambda, \operatorname{div} v\right) \quad \forall v \in V_{h} .
$$

Therefore, using (3.14) and again a well-known result from approximation theory [5], we obtain

$$
|(\lambda-\tilde{\lambda}, \operatorname{div} v)| \leqslant C \mathcal{F C}\left\|\lambda-\pi_{h} \lambda\right\|_{0} \leqslant C_{1} \mathcal{F C h}|\lambda|_{1} .
$$

Finally, to estimate the third term on the right-hand side of (3.16), let $\mu=$ $\Sigma_{i j k} \alpha_{i j k} \xi_{i j k}=\Sigma_{k} \mu_{k}$. Using (3.9), (3.14), and (3.17), we see that

$$
\left(\sum_{k=1}^{3} \mu_{k}, \operatorname{div}(u-\tilde{u})\right) \leqslant C|\mu|_{h}|u-\tilde{u}|_{1} \leqslant C_{1} h|u|_{2} .
$$

To estimate the remaining term $\left(\mu_{4}, \operatorname{div}(u-\tilde{u})\right)$, we recall that $\lambda_{h}$ and $\tilde{\lambda} \in N_{h}^{\perp}$ so that $\mu=\lambda_{h}-\tilde{\lambda} \in N_{h}^{\perp}$. Hence $\Sigma_{i, j}^{4} \alpha_{i j 4}=0$, and thus by Lemma 3.3 and (3.14) we have

$$
\left\|\mu_{4}\right\|_{0, q} \leqslant C(q) h^{-1}|\mu|_{h} \leqslant C_{1}(q) \mathcal{H} h^{-1}, \quad q<\infty .
$$

Thus, using Hölder's inequality, we find that

$$
\left|\left(\mu_{4}, \operatorname{div}(u-\tilde{u})\right)\right| \leqslant C \Gamma_{p}(u-\tilde{u})\left\|\mu_{0}\right\|_{0, q} \leqslant C_{1}(p) \mathcal{S C}^{-1} \Gamma_{p}(u-\tilde{u}),
$$

for $p>1,1 / q+1 / p=1$, where

$$
\begin{aligned}
\Gamma_{p}(v) & =h^{2 / p-2}\left\{\sum_{i, j}\left|\int_{K_{i j}} \xi_{i j 4} \operatorname{div} v d x d y\right|^{p}\right\}^{1 / p} \\
& \leqslant C h^{2 / p-2}\left\{\sum_{\Delta \in \mathcal{C}_{h}}\left|\int_{\Delta} \operatorname{div} v d x d y\right|^{p}\right\}^{1 / p} .
\end{aligned}
$$


To estimate $\Gamma_{p}(u-\tilde{u})$ we shall use the following "superapproximation" result. Here and below $P_{k}$ denotes the set of polynomials in $x$ and $y$ of degree at most $k$.

Lemma 3.4. Defining for $v \in H^{1}(\Delta), \Delta \in \bigodot_{h}$,

$$
L(v)=\int_{\Delta} \frac{\partial}{\partial x}(v-\tilde{v}) d x d y,
$$

where $\tilde{v}$ is the bilinear function interpolating $v$ at the corners of the rectangle $\Delta$, we have $L(v)=0$ for $v \in P_{2}$, so that for $1 \leqslant p<\infty$,

$$
|L(v)| \leqslant C h^{4-2 / p}|v|_{W^{3, p}(\Delta)} .
$$

An analogous estimate holds with $\partial / \partial x$ replaced by $\partial / \partial y$.

From Lemma 3.4 we conclude that

$$
\Gamma_{p}(u-\tilde{u}) \leqslant C h^{2}|u|_{3, p},
$$

which together with (3.19) and (3.20) shows that for $p>1$

$$
|(\mu, \operatorname{div}(u-\tilde{u}))| \leqslant C \mathcal{H C} h|u|_{2}+C(p) \mathcal{H C} h|u|_{3, p} \text {. }
$$

Estimating the right-hand side of (3.16), using (3.17), (3.18), and (3.21), and combining the result with (3.12) and (3.15) we find that for $p>1$

$$
\begin{aligned}
\left|u_{h}-\tilde{u}\right|_{1}+\left|\lambda_{h}-\tilde{\lambda}\right|_{h}+\sqrt{\varepsilon}\left\|\lambda_{h}-\tilde{\lambda}\right\|_{0} \\
\quad \leqslant C h\left(|u|_{2}+|\lambda|_{1}\right)+C(p) h|u|_{3, p}+C h\|\lambda\|_{0} .
\end{aligned}
$$

Using finally the triangle inequality recalling (3.17), we obtain the desired estimate for $\left|u-u_{h}\right|_{1}+\left|\lambda_{h}-\tilde{\lambda}\right|_{h}$, and the proof of Theorem 3.1 is complete.

3.3. Smoothing of the Pressure. Since by (3.8) we only have $\|\mu\|_{0} \leqslant C h^{-1}|\mu|_{h}$ for $\mu \in N_{h}^{\perp}$, we cannot from Theorem 3.1 conclude any convergence rate in $L_{2}(\Omega)$ for the pressure $\lambda_{h}$. However, by filtering out the component $\lambda_{h 4}$ by a simple smoothing procedure one can obtain $\theta(h)$-convergence for the smoothed pressure. As an example of such a smoothing procedure we may take the $L_{2}$-projection $\pi_{h}^{1}$ of $Q_{h}$ onto $Q_{h}^{1}$

$$
\pi_{h}^{1} \lambda_{h}(x, y)=\frac{1}{4} \sum_{k=1}^{4} \lambda_{i j k}, \quad(x, y) \in K_{i j} \in \mathcal{C}_{h}^{0},
$$

where $\lambda_{i j k}, k=1, \ldots, 4$, denotes the value of $\lambda_{h}$ on the four subrectangles of $K_{i j}$.

COROLlaRY 3.1. Under the assumptions of Theorem 3.1, we have

$$
\left\|\lambda-\pi_{h}^{1} \lambda_{h}\right\|_{0} \leqslant C(p) h\left(|u|_{2}+|u|_{3, p}+\|\lambda\|_{1}\right) \text {. }
$$

Proof. Recalling (3.9), we have, by Theorem 3.1,

$$
\left\|\pi_{h}^{1} \lambda_{h}-\pi_{h}^{1} \tilde{\lambda}\right\|_{0} \leqslant\left|\lambda_{h}-\tilde{\lambda}\right|_{h} \leqslant C h\left(|u|_{2}+|u|_{3, p}+\|\lambda\|_{1}\right) \text {. }
$$

Further, since $(\lambda, 1)=0$, we have $\pi_{h}^{1} \tilde{\lambda}=\pi_{h}^{1} \lambda$. Together with the classical estimate $\left\|\lambda-\pi_{h}^{1} \lambda\right\|_{0} \leqslant C h|\lambda|_{1}$, this proves the desired estimate.

Remark. The nonconvergence in $L_{2}(\Omega)$ of the pressures $\lambda_{h}$ has been observed in practice, cf. [13], where also smoothing is discussed. 
3.4. An $L_{2}$-Estimate for the Velocities. We shall now prove an error-estimate for the velocities in the $L_{2}$-norm. We shall not use the standard duality argument here since this would give a weaker estimate than that proved below but instead base the argument on another stability estimate related to the method (3.3). To state this estimate, we need some additional mesh-dependent norms; see [2]. Let

$$
H^{2, h}(\Omega)=\left\{v \in H_{0}^{1}(\Omega): v_{\mid \Delta} \in H^{2}(\Omega), \Delta \in \mathcal{C}_{h}\right\},
$$

and define on $H^{2, h}(\Omega)$ the seminorm $|\cdot|_{2, h}$ as follows:

$$
|v|_{2, h}^{2}=\sum_{\Delta \in \mathcal{C}_{h}}|v|_{H^{2}(\Delta)}^{2}+h^{-1} \sum_{\Delta_{1}, \Delta_{2} \in \mathcal{C}_{h}} \int_{S_{12}}\left(\frac{\partial v}{\partial \nu_{1}}+\frac{\partial v}{\partial \nu_{2}}\right)^{2} d s,
$$

where $\nu_{i}$ denotes the unit normal to the common boundary $S_{12}$ of $\Delta_{1}$ and $\Delta_{2}$ exterior to $\Delta_{i}$. Further, we define on $H_{0}^{1}(\Omega)$ a norm $\|\cdot\|_{0, h}$ by

$$
\|v\|_{0, h}^{2}=\|v\|_{0}^{2}+h \sum_{\Delta_{1}, \Delta_{2} \in \mathcal{C}_{h}} \int_{S_{12}} v^{2} d s .
$$

For vector functions $v=\left(v_{1}, v_{2}\right)$, we set as usual

$$
|v|_{2, h}^{2}=\left|v_{1}\right|_{2, h}^{2}+\left|v_{2}\right|_{2, h}^{2} \text { and }\|v\|_{0, h}^{2}=\left\|v_{1}\right\|_{0, h}^{2}+\left\|v_{2}\right\|_{0, h}^{2} \text {. }
$$

We recall (see [2]) that $|\cdot|_{2, h}$ is actually a norm on $H^{2, h}(\Omega)$ and that

$$
\begin{aligned}
& (\nabla u, \nabla v) \leqslant\|u\|_{0, h}|v|_{2, h}, \quad u \in H_{0}^{1}(\Omega), \quad v \in H^{2, h}(\Omega), \\
& |v|_{2, h} \leqslant C h^{-1}|v|_{1}, \quad v \in V_{h}, \\
& \|v\|_{0, h} \leqslant C\|v\|_{0}, \quad v \in V_{h} \text {. }
\end{aligned}
$$

Let us now introduce the subspace $V_{h}^{0} \subset V_{h}$ defined by

$$
V_{h}^{0}=\left\{v \in V_{h} ;(\mu, \operatorname{div} v)=0 \forall \mu \in Q_{h}^{1}\right\} .
$$

The stability estimate, which we will need below, is the following

LEMMA 3.5. There is a constant $C$ such that

$$
\sup _{v \in V_{h}^{0}} \frac{(\nabla u, \nabla v)}{|v|_{2, h}} \geqslant C\|u\|_{0} \quad \forall u \in V_{h}^{0} .
$$

Proof. Given $u \in V_{h}^{0}$, let $(z, \nu) \in\left[H_{0}^{1}(\Omega)\right]^{2} \times L_{2}(\Omega)$ be the solution of the problem

$$
\begin{cases}-\Delta z+\Delta \nu=u & \text { in } \Omega \\ \operatorname{div} z=0 & \text { in } \Omega \\ (\nu, 1)=0 & \end{cases}
$$

and let $\left(z_{h}, \nu_{h}\right) \in V_{h} \times Q_{h}^{1}$ be an approximation of $(z, \nu)$ defined by

$$
\begin{cases}\left(\nabla z_{h}, \nabla v\right)-\left(\nu_{h}, \operatorname{div} v\right)=(u, v) & \forall v \in V_{h}, \\ \left(\mu, \operatorname{div} z_{h}\right)=0 & \forall \mu \in Q_{h}^{1}, \\ \left(\nu_{h}, 1\right)=0 & \end{cases}
$$

Then we have $z_{h} \in V_{h}^{0}$ and

$$
\left(\nabla u, \nabla z_{h}\right)=\|u\|_{0}^{2} .
$$


To estimate the error $\left|z-z_{h}\right|_{1}$, we note that by Lemma 3.2 the mixed method defined by (3.25) is uniformly stable in the "classical" sense (cf. [1], [4]). Recalling (3.7), we thus have the quasioptimal estimate

$$
\left|z-z_{h}\right|_{1} \leqslant C h\left(|z|_{2}+|\nu|_{1}\right) \leqslant C_{1} h\|u\|_{0} .
$$

Further, using (3.23), (3.27), and the approximation results of [2], we obtain, with $\tilde{z} \in V_{h}$ being the interpolant of $z$,

$$
\begin{aligned}
\left|z_{h}\right|_{2, h} & \leqslant\left|z_{h}-\tilde{z}\right|_{2, h}+|z-\tilde{z}|_{2, h}+|z|_{2, h} \\
& \leqslant C h^{-1}\left|z_{h}-\tilde{z}\right|_{1}+C|z|_{2} \leqslant C_{1}\left(|z|_{2}+|\nu|_{1}\right) \leqslant C_{2}\|u\|_{0} .
\end{aligned}
$$

Together with (3.26), this proves the lemma.

We can now prove the $L_{2}$-estimate for the velocities.

THEOREM 3.2. Under the assumptions of Theorem 3.1, we have

$$
\left\|u-u_{h}\right\|_{0} \leqslant C(p) h^{2}\left(|u|_{2}+|u|_{3, p}+\|\lambda\|_{1}\right) .
$$

Proof. Let $\left(z_{h}, v_{h}\right) \in V_{h} \times Q_{h}^{1}$ be another approximation to the solution $(u, \lambda)$ of (3.2) defined by

$$
\begin{cases}\left(\nabla z_{h}, \nabla v\right)-\left(\nu_{h}, \operatorname{div} v\right)=(f, v) & \forall v \in V_{h}, \\ \left(\mu, \operatorname{div} z_{h}\right)=-\varepsilon\left(\lambda_{h}, \mu\right) & \forall \mu \in Q_{h}^{1}, \\ \left(v_{h}, 1\right)=0 . & \end{cases}
$$

Since $\left(\lambda_{h}, 1\right)=0$, it follows from Lemma 3.2 that this problem has a unique solution. More precisely, by the argument leading to (3.27) together with the usual duality argument we have with $z_{h}^{0}$ being the solution of (3.28) with $\varepsilon=0$ :

$$
\left\|u-z_{h}^{0}\right\|_{0} \leqslant C h^{2}\left(|u|_{2}+|\lambda|_{1}\right) \text {. }
$$

Further, by linearity and using once again Lemma 3.2, we conclude that writing $w_{h}=z_{h}-z_{h}^{0}$

$$
\left\|w_{h}\right\|_{1} \leqslant C \sup _{\mu \in Q_{h}^{\prime}} \frac{\left|\varepsilon\left(\lambda_{h}, \mu\right)\right|}{\|\mu\|_{0}} \leqslant C_{1} \varepsilon\left|\lambda_{h}\right|_{h} \leqslant C_{2} h^{2}\left(\|\lambda\|_{0}+\left|\lambda_{h}-\tilde{\lambda}\right|_{h}\right),
$$

which shows that

$$
\left\|u-z_{h}\right\|_{0} \leqslant C h^{2}\left(|u|_{2}+\|\lambda\|_{1}+\left|\lambda_{h}-\tilde{\lambda}\right|_{h}\right) .
$$

Next, let us combine (3.3a) and (3.2) to obtain

$$
\begin{aligned}
& \left(\nabla\left(u_{h}-z_{h}\right), \nabla v\right) \\
& \quad=\left(\nabla\left(u-z_{h}\right), \nabla v\right)+\left(\lambda_{h}-\tilde{\lambda}, \operatorname{div} v\right)-(\lambda-\tilde{\lambda}, \operatorname{div} v) \quad \forall v \in V_{h} .
\end{aligned}
$$

Note that, by (3.3b) and (3.28), we have $u_{h}-z_{h} \in V_{h}^{0}$. Therefore, we may apply Lemma 3.5 to (3.30) to obtain

$$
\begin{aligned}
\left\|u_{h}-z_{h}\right\|_{0} \leqslant C \sup _{\substack{v \in V_{h}^{0} \\
|v|_{2, h}=1}}\left\{\left(\nabla\left(u-z_{h}\right), \nabla v\right)+\right. & \left(\lambda_{h}-\tilde{\lambda}, \operatorname{div} v\right) \\
& -(\lambda-\tilde{\lambda}, \operatorname{div} v)\} .
\end{aligned}
$$


Let us now estimate the right-hand side of (3.31). First we notice that if $\tilde{u} \in V_{h}$ is the interpolant of $u$, we have, by (3.22) and (3.24),

$$
\begin{aligned}
& \left(\nabla\left(u-z_{h}\right), \nabla v\right) \leqslant\left\|u-z_{h}\right\|_{0, h}|v|_{2, h} \leqslant\left(\|u-\tilde{u}\|_{0, h}+\left\|z_{h}-\tilde{u}\right\|_{0, h}\right)|v|_{2, h} \\
& \quad \leqslant\left(\|u-\tilde{u}\|_{0, h}+C\left\|z_{h}-\tilde{u}\right\|_{0}\right)|v|_{2, h} \leqslant C_{1}\left(\|u-\tilde{u}\|_{0, h}+\left\|u-z_{h}\right\|_{0}\right)|v|_{2, h} .
\end{aligned}
$$

By [2] we have

$$
\|u-\tilde{u}\|_{0, h} \leqslant C h^{2}|u|_{2},
$$

and thus, recalling (3.29), we conclude that

$$
\left(\nabla\left(u-z_{h}\right), \nabla v\right) \leqslant C h^{2}\left(|u|_{2}+\|\lambda\|_{1}+\left|\lambda_{h}-\tilde{\lambda}\right|_{h}\right)|v|_{2, h} \text {. }
$$

Next, to estimate the second term on the right side of (3.31), let us write

$$
\lambda_{h}-\tilde{\lambda}=\sum_{i j k} \alpha_{i j k} \xi_{i j k}=\sum_{k=1}^{4} \mu_{k}
$$

so that

$$
\left(\lambda_{h}-\tilde{\lambda}, \operatorname{div} v\right)=\sum_{k=2}^{4}\left(\mu_{k}, \operatorname{div} v\right), \quad v \in V_{h}^{0} .
$$

Consider now a given $K_{i j} \in \mathcal{C}_{h}^{0}$. Let $\Delta_{k} \in \mathcal{C}_{h}, k=1, \ldots, 4$, be the four subrectangles of $K_{i j}$ and define

$$
|v|_{H^{2, h}\left(K_{i j}\right)}^{2}=\sum_{k}|v|_{H^{2}\left(\Delta_{k}\right)}^{2}+h^{-1} \sum_{m=1}^{2} \sum_{k, l} \int_{S_{k l}}\left(\frac{\partial v_{m}}{\partial \nu_{k}}+\frac{\partial v_{m}}{\partial \nu_{l}}\right)^{2} d s .
$$

We note that if $v \in V_{h}$, then $|v|_{H^{2, h}\left(K_{i j}\right)}=0$ if and only if $v_{\mid K_{i j}} \in\left[P_{1}\left(K_{i j}\right)\right]^{2}$. Together with the fact that

$$
\int_{K_{i j}} \xi_{i j k} \operatorname{div} v d x d y=0 \quad \text { for } k=2,3, v \in\left[P_{1}\left(K_{i j}\right)\right]^{2},
$$

we conclude via a scaling argument that for $k=2,3, v \in V_{h}$,

$$
\left|\int_{K_{i j}} \xi_{i j k} \operatorname{div} v d x d y\right| \leqslant C h^{2}|v|_{H^{2, h}\left(K_{i j}\right)}, \quad k=2,3, v \in V_{h}, K_{i j} \in C_{h}^{0} .
$$

Thus, for $k=2,3, v \in V_{h}$ we have

$$
\left(\mu_{k}, \operatorname{div} v\right) \leqslant C h\left\|\mu_{k}\right\|_{0}\left\{\sum_{i j}|v|_{H^{2, h}\left(K_{i j}\right)}^{2}\right\}^{1 / 2} \leqslant C h\left\|\mu_{k}\right\|_{0}|v|_{2, h} .
$$

Finally, to estimate $\left(\mu_{4}, \operatorname{div} v\right)$ we recall that (3.11) holds for any $\mu_{4}=\Sigma_{i j} \alpha_{i j 4} \xi_{i j 4}$ and $g \in V_{h}$. Therefore, applying the easy-to-check inequality

$$
\begin{gathered}
|v|_{2, h}^{2} \geqslant C h^{-2} \sum_{i=1}^{2 n-1} \sum_{j=1}^{2 m-1} \sum_{k=1}^{2}\left[\left(v_{k, i-1, j}-2 v_{k i j}+v_{k, i+1, j}\right)^{2}\right. \\
\left.+\left(v_{k i, j-1}-2 v_{k i j}+v_{k i, j+1}\right)^{2}\right], \\
v=\left(v_{1}, v_{2}\right) \in V_{h}, \quad v_{k i j}=v_{k}\left(i h_{1} / 2, j h_{2} / 2\right),
\end{gathered}
$$

we obtain

$$
\left(\mu_{4}, \operatorname{div} v\right) \leqslant C h^{2} \sigma\left(\dot{\mu}_{4}\right)|v|_{2, h}, \quad v \in V_{h}
$$


Combining now the estimates for $\left(\mu_{k}, \operatorname{div} v\right), k=2,3,4$, we find that

$$
\left(\lambda_{h}-\tilde{\lambda}, \operatorname{div} v\right) \leqslant C h\left|\lambda_{h}-\tilde{\lambda}\right|_{h}|v|_{2, h}, \quad v \in V_{h}^{0} .
$$

We finally have to estimate the term $(\lambda-\tilde{\lambda}, \operatorname{div} v)$. To this end we first note that if $\pi_{h}$ is the orthogonal projection of $L_{2}(\Omega)$ onto $Q_{h}$, then

$$
\left(\operatorname{div} v-\pi_{h} \operatorname{div} v\right)_{\mid \Delta} \equiv 0 \quad \text { if } v_{\mid \Delta} \in\left[P_{1}(\Delta)\right]^{2}, \Delta \in \mathcal{C}_{h},
$$

so that by a scaling argument

$$
\left\|\operatorname{div} v-\pi_{h} \operatorname{div} v\right\|_{L_{2}(\Delta)} \leqslant C h|v|_{H^{2}(\Delta)}, \quad \Delta \in \mathcal{C}_{h}, v \in V_{h} .
$$

Thus, for $v \in V_{h}$ we have

$$
\begin{aligned}
(\lambda-\tilde{\lambda}, \operatorname{div} v) & =\left(\lambda-\pi_{h} \lambda, \operatorname{div} v\right)=\left(\lambda-\pi_{h} \lambda, \operatorname{div} v-\pi_{h} \operatorname{div} v\right) \\
& \leqslant\left\|\lambda-\pi_{h} \lambda\right\|_{0}\left\|\operatorname{div} v-\pi_{h} \operatorname{div} v\right\|_{0} \\
& \leqslant C h^{2}|\lambda|_{1}\left\{\sum_{\Delta \in e_{h}}|v|_{H^{2}(\Delta)}^{2}\right\}^{1 / 2} \leqslant C h^{2}|\lambda|_{1}|v|_{2, h} .
\end{aligned}
$$

Combining now (3.32)-(3.34), we get

$$
\left\|u_{h}-z_{h}\right\|_{0} \leqslant C h^{2}\left(|u|_{2}+|\lambda|_{1}\right)+C h\left|\lambda_{h}-\tilde{\lambda}\right|_{h} .
$$

Recalling finally (3.29) and the estimate already proved for $\left|\lambda_{h}-\tilde{\lambda}\right|_{h}$ in Theorem 3.1, we obtain the stated estimate for $\left\|u-u_{h}\right\|_{0}$ and the proof is complete.

Remark. Comparing the original problem (3.3) and the "simplified" problem (3.28) obtained by replacing $Q_{h}$ by $Q_{h}^{1}$, we note that we have the same rate of convergence in the two cases. However, after eliminating the pressures (3.28) results in a positive definite matrix equation with bandwidth twice as big as that obtained from (3.3). Thus, the "simplified" problem may in fact be more costly to solve numerically.

4. A Mixed Method for a Plate Problem. The biharmonic problem

$$
\left\{\begin{array}{l}
\Delta^{2} u=f \\
u=H_{0}^{2}(\Omega)
\end{array}\right.
$$

can be given the following variational formulation:

$$
\operatorname{Inf}_{v \in H_{0}^{2}(\Omega)}\left\{\frac{1}{2} \int_{\Omega}\left[\left(\frac{\partial^{2} v}{\partial x^{2}}\right)^{2}+2\left(\frac{\partial^{2} v}{\partial x \partial y}\right)^{2}+\left(\frac{\partial^{2} v}{\partial y^{2}}\right)^{2}\right] d x d y-\int_{\Omega} f v d x d y\right\}
$$

The solution of (4.2) satisfies (4.1) and vice versa. Introducing the auxiliary variable $\varphi=\left(\varphi_{1}, \varphi_{2}\right)=\nabla v$, we can formulate (4.2) as follows:

$$
\operatorname{Inf}_{\substack{(v, \varphi) \in V \times V^{2} \\ \varphi=\nabla v}}\left\{\frac{1}{2}\|\nabla \varphi\|^{2}-(f, v)\right\},
$$

where $V=H_{0}^{1}(\Omega)$ and

$$
\|\nabla \varphi\|^{2}=\left\|\nabla \varphi_{1}\right\|_{0}^{2}+\left\|\nabla \varphi_{2}\right\|_{0}^{2}, \quad \varphi=\left(\varphi_{1}, \varphi_{2}\right) .
$$


Enforcing here the side condition $\varphi=\nabla v$ approximately via a penalty term, we are led to the following minimization problem:

$$
\operatorname{Inf}_{(v, \varphi) \in V \times V^{2}}\left\{\frac{1}{2}\|\nabla \varphi\|^{2}+\frac{1}{2 \varepsilon}\|\varphi-\nabla v\|_{0}^{2}-(f, v)\right\} .
$$

Below we shall consider a discrete analogue of this problem.

Remark 4.1. The problem (4.4) corresponds in fact to the simplest model for a moderately thin plate with thickness $\varepsilon$, taking shear deformations into account. We clearly obtain (4.3) as limit problem from (4.4) as $\varepsilon$ tends to zero. In most practical problems $\varepsilon$ is not very small, and then (4.4) is a much better model for a plate than (4.3). Below, we shall only consider the case when $\varepsilon$ is very small and compare the solution of the discrete problem with the exact solution of (4.3). However, it is also possible to compare the discrete solution with the solution of (4.4) without extra complications. In fact (4.4) becomes more "well-conditioned" from a numerical point of view as $\varepsilon / h$ increases; if $\varepsilon / h$ is (moderately) large one can apply a standard finite element method to (4.4), replacing $V$ by a finite-dimensional subspace, and obtain good results.

Let us now introduce the following discrete analogue of (4.4) stated in [11]:

$$
\operatorname{Inf}_{(v, \varphi) \in V_{h} \times W_{h}}\left\{\frac{1}{2}\|\nabla \varphi\|^{2}+\frac{1}{2 \varepsilon}(\varphi-\nabla v, \varphi-\nabla v)_{*}-(f, v)\right\},
$$

where $V_{h}=S_{h}, W_{h}=S_{h}^{2}$ and, as above, the middle term is evaluated using one-point Gaussian quadrature. The corresponding discrete analogue with exact evaluation of this term will be a useless model if $\varepsilon / h \lesssim 1$.

The problem (4.5) can also be formulated as the following saddle-point problem:

$$
\operatorname{Inf}_{(v, \varphi) \in V_{h} \times W_{h}} \operatorname{Sup}_{\mu \in Q_{h}}\left\{\frac{1}{2}\|\nabla \varphi\|^{2}+(\varphi-\nabla v, \mu)-\frac{\varepsilon}{2}\|\mu\|_{0}^{2}-(f, v)\right\},
$$

where now $Q_{h}=T_{h}^{2}$. The condition for $\left(u_{h}, \theta_{h}, \lambda_{h}\right) \in V_{h} \times W_{h} \times Q_{h}$ to be a saddle-point for the problem (4.6) reads

$$
\begin{cases}\left(\nabla \theta_{h}, \nabla \varphi\right)+\left(\lambda_{h}, \varphi\right)=0 & \forall \varphi \in W_{h}, \\ -\left(\lambda_{h}, \nabla v\right)=(f, v) & \forall v \in V_{h}, \\ \varepsilon\left(\lambda_{h}, \mu\right)-\left(\theta_{h}-\nabla u_{h}, \mu\right)=0, & \forall \mu \in Q_{h} .\end{cases}
$$

This is the discrete problem to be analyzed below. Let us note that the continuous analogue of (4.7) reads

$$
\left\{\begin{array}{l}
-\Delta \theta+\lambda=0 \\
\operatorname{div} \lambda=f \\
\varepsilon \lambda-\theta+\nabla u=0 \\
(u, \theta) \in V \times V^{2}
\end{array}\right.
$$

If we here take $\varepsilon=0$ and eliminate $\theta$ and $\lambda$, we obtain the biharmonic problem (4.1). Thus, the discrete model (4.7) to be studied can be considered to be a mixed method for the biharmonic problem obtained starting from the formulation (4.8) (with $\varepsilon=0$ ). Also, (4.8) is a model for a moderately thin plate with thickness $\varepsilon$ and $u, \theta$ and $\lambda$ being the vertical displacement, rotations and shear forces, respectively. 
Let us now analyze the method (4.7). First, we note that, taking $(v, \varphi, \mu)=$ $\left(u_{h}, \theta_{h}, \lambda_{h}\right)$ in (4.7), we obtain

$$
\left|\theta_{h}\right|_{1}^{2}+\varepsilon\left\|\lambda_{h}\right\|_{0}^{2}=\left(f, u_{h}\right) .
$$

Further, it is easy to see that (4.7c) determines $u_{h}$ uniquely in terms of $\theta_{h}$ and $\lambda_{h}$. Hence solutions of (4.7) are uniquely determined, and thus also existence of a solution of (4.7) follows.

Next, let us introduce the orthogonal basis $\left\{\eta_{i j k}\right\}, i=1, \ldots, n, j=1, \ldots, m$, $k=1, \ldots, 8$, for the space $Q_{h}=T_{h}^{2}$ defined as follows:

$$
\begin{array}{ll}
\eta_{i j 1}=\left(\xi_{i j 1}, 0\right), & \eta_{i j 2}=\left(0, \xi_{i j 1}\right), \\
\eta_{i j 3}=\left(b \xi_{i j 2}, a \xi_{i j 3}\right), & \eta_{i j 4}=\left(-\xi_{i j 3}, 0\right), \\
\eta_{i j 5}=\left(0, \xi_{i j 2}\right), & \eta_{i j 6}=\left(-\xi_{i j 4}, 0\right), \\
\eta_{i j 7}=\left(0, \xi_{i j 4}\right), & \eta_{i j 8}=\left(a \xi_{i j 2},-b \xi_{i j 3}\right),
\end{array}
$$

where $\xi_{i j k} \in T_{h}$ are the basis functions introduced in Section 3 and

$$
a=2 h_{1} /\left(h_{1}+h_{2}\right), \quad b=2 h_{2} /\left(h_{1}+h_{2}\right) .
$$

The basis functions $\eta_{i j k} \equiv\left(\eta_{i j k, 1}, \eta_{i j k, 2}\right)$ take values on $K_{i j}$ according to Figure 2 and are zero outside $K_{i j}$.

$$
\begin{aligned}
& k \quad 1 \\
& \begin{array}{|l|l|l|}
\hline \eta_{i j k, 1} & 1 & 1 \\
\hline 1 & 1 \\
\hline
\end{array} \\
& \begin{array}{|l|l|}
\hline 0 & 0 \\
\hline 0 & 0 \\
\hline
\end{array} \\
& 3 \\
& \begin{array}{|l|l|}
\hline b & -b \\
\hline b & -b \\
\hline
\end{array} \\
& 4 \\
& \begin{array}{|l|l|l|}
\hline \eta_{i j k, 2} & 0 & 0 \\
\hline 0 & 0 \\
\hline
\end{array} \\
& k \\
& 5 \\
& \begin{array}{l|l|l|}
\hline & \eta_{i j k, 1} \quad \begin{array}{l|l}
0 \\
0
\end{array} & 0 \\
\hline 0 & 0 \\
\hline
\end{array} \\
& \begin{array}{|l|l|}
\hline 1 & 1 \\
\hline 1 & 1 \\
\hline
\end{array} \\
& \begin{array}{|r|r|}
\hline-a & -a \\
\hline a & a \\
\hline
\end{array} \\
& 7 \\
& \begin{array}{|r|r|}
\hline 1 & -1 \\
\hline-1 & 1 \\
\hline
\end{array} \\
& \begin{array}{|l|l|}
\hline 0 & 0 \\
\hline 0 & 0 \\
\hline
\end{array} \\
& \begin{array}{r|r}
1 & 1 \\
\hline-1 & -1
\end{array} \\
& \begin{array}{l|l|l|}
\hline & \eta_{i j k, 2} & -1 \\
\hline 1 & -1 \\
\hline
\end{array} \\
& \begin{array}{|l|l|}
\hline 0 & 0 \\
\hline 0 & 0 \\
\hline
\end{array} \\
& \begin{array}{|l|l|}
\hline a & -a \\
\hline a & -a \\
\hline
\end{array}
\end{aligned}
$$
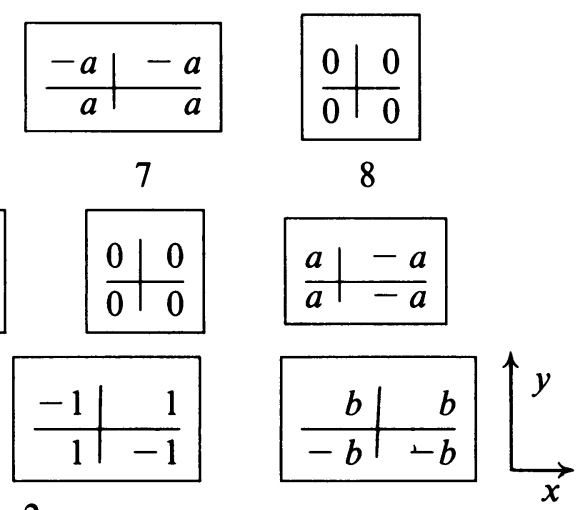

Figure 2

The local basis functions of $Q_{h}$

Let us now introduce

$$
\begin{aligned}
& N_{h}=\left\{\lambda \in Q_{h}:(\lambda, \varphi-\nabla v)=0 \forall v \in V_{h}, \forall \varphi \in W_{h}\right\}, \\
& N_{h}^{\perp}=\left\{\lambda \in Q_{h}:(\lambda, \mu)=0 \forall \mu \in N_{h}\right\} .
\end{aligned}
$$

It is easy to see that $N_{h}$ contains the functions $\rho_{i}, i=1, \ldots, 2 n$, and $\omega_{j}, j=1, \ldots, 2 m$, where

$$
\rho_{i}(x, y)= \begin{cases}\left((-1)^{j}, 0\right) & \text { if }(x, y) \in \Delta_{i j} \in C_{h}, 1 \leqslant j \leqslant 2 m \\ (0,0) & \text { otherwise, }\end{cases}
$$


and

$$
\omega_{j}(x, y)= \begin{cases}\left(0,(-1)^{i}\right) & \text { if }(x, y) \in \Delta_{i j} \in \mathcal{C}_{h}, 1 \leqslant i \leqslant 2 n, \\ (0,0) & \text { otherwise. }\end{cases}
$$

Any $\lambda \in N_{h}$ can then be represented as

$$
\lambda=\sum_{i=1}^{2 n} \alpha_{i} \rho_{i}+\sum_{j=1}^{2 m} \beta_{j} \omega_{j}+r,
$$

where $\alpha_{i}, \beta_{j} \in \mathbf{R}$ and $r=\left(r_{1}, r_{2}\right)$ satisfies

$$
\begin{array}{ll}
r_{1}(x, y)=0 & \text { if }(x, y) \in \Delta_{i 1} \in \mathcal{C}_{h}, 1 \leqslant i \leqslant 2 n, \\
r_{2}(x, y)=0 & \text { if }(x, y) \in \Delta_{1 j} \in \mathcal{C}_{h}, 1 \leqslant j \leqslant 2 m .
\end{array}
$$

A simple computation shows that $r=C \bar{\varphi}$, where $C$ is a constant and

$$
\begin{aligned}
\bar{\varphi}(x, y)=\left((-1)^{i+j}(j-1) a,(-1)^{i+j+1}(i-1) b\right), \\
(x, y) \in \Delta_{i j} \in \mathcal{C}_{h}, 1 \leqslant i \leqslant 2 n, 1 \leqslant j \leqslant 2 m .
\end{aligned}
$$

Thus, $N_{h}$ is the $(2 n+2 m+1)$-dimensional space spanned by the functions $\rho_{i}, \omega_{j}$, and $\bar{\varphi}$. Using this characterization of $N_{h}$, it is easy to verify that $\lambda=\Sigma_{i, j, k} \alpha_{i j k} \eta_{i j k}$ belongs to $N_{h}^{\perp}$ if and only if

$$
\left\{\begin{array}{l}
\sum_{j} \alpha_{i j k}=0, \quad k=4,6,1 \leqslant i \leqslant n \\
\sum_{i} \alpha_{i j k}=0, \quad k=5,7,1 \leqslant j \leqslant m
\end{array}\right.
$$

and

$$
4 a \sum_{i, j} j \alpha_{i j 6}+4 b \sum_{i, j} i \alpha_{i j 7}+2\left(a^{2}+b^{2}\right) \sum_{i, j} \alpha_{i j 8}=0 .
$$

Let us now introduce the following mesh-dependent norm on $Q_{h}$ :

$$
\begin{gathered}
\|\mu\|_{h}=\left\{h^{4} \sum_{k=1}^{3} \sum_{i, j}\left(\alpha_{i j k}\right)^{2}+h^{6} \sum_{k=4,5,8} \sum_{i, j}\left(\alpha_{i j k}\right)^{2}+h^{8} \sum_{k=6,7} \sum_{i, j}\left(\alpha_{i j k}\right)^{2}\right\}^{1 / 2}, \\
\mu=\sum_{i, j, k} \alpha_{i j k} \eta_{i j k} \in Q_{h} .
\end{gathered}
$$

Comparing this norm with the $L_{2}$-norm, we see that

$$
C_{1} h^{3}\|\mu\|_{0} \leqslant\|\mu\|_{h} \leqslant C h\|\mu\|_{0} \quad \forall \mu \in Q_{h} .
$$

In the proof of the error estimates below we shall use the following three lemmas:

LEMMA 4.1. There is a positive constant $C$ such that, for all $\mu \in N_{h}^{\perp}$,

$$
\sup _{(v, \varphi) \in V_{h} \times W_{h}} \frac{(\mu, \varphi-\nabla v)}{\|\varphi\|_{1}+h^{-1}\|v\|_{1}} \geqslant C\|\mu\|_{h} .
$$


Proof. Let $\mu=\sum_{i, j, k} \alpha_{i j k} \eta_{i j k} \in N_{h}^{\perp}$ be given, and define

$$
\begin{array}{rlrl}
\mu_{k} & =\sum_{i, j} \alpha_{i j k} \eta_{i j k}, & k=1, \ldots, 8, \\
\beta & =\frac{1}{n m} \sum_{i, j} \alpha_{i j 8}, & \tilde{\alpha}_{i j 8}=\alpha_{i j 8}-\beta, \\
\mu_{80}=\beta \sum_{i, j} \eta_{i j 8}, & \mu_{81}=\mu_{8}-\mu_{80},
\end{array}
$$

so that in particular

$$
\mu_{81}=\sum_{i, j} \tilde{\alpha}_{i j 8} \eta_{i j 8}, \quad \sum_{i, j} \tilde{\alpha}_{i j 8}=0 .
$$

Next, let us define the functions $z, w \in V_{h}$ and $\chi, \zeta \in W_{h}$ as follows:

$$
\begin{gathered}
\begin{cases}\chi_{1}(P)=h^{2} \alpha_{i j 1} \\
\chi_{2}(P)=h^{2} \alpha_{i j 2} \quad \text { if } P \text { is the midpoint of } K_{i j} \in \mathcal{C}_{h}^{0} ; \\
z(P)=h^{3} \alpha_{i j 3}\end{cases} \\
\begin{cases}\zeta_{1}(P)=h^{2}\left(\alpha_{i+1, j 8}-\alpha_{i j 8}\right) & \text { if } P \text { is the midpoint } \\
\zeta_{2}(P)=h^{2}\left(\alpha_{i+1, j 5}-\alpha_{i j 5}\right) & \text { of the common side of } \\
w(P)=h^{5}\left(\alpha_{i+1, j 7}-\alpha_{i j 7}-4 b \beta\right) & K_{i j}, K_{i+1, j} \in \mathcal{C}_{h}^{0} ;\end{cases} \\
\begin{cases}\zeta_{1}(P)=h^{2}\left(\alpha_{i j 4}-\alpha_{i, j+1,4}\right) & \text { if } P \text { is the midpoint } \\
\zeta_{2}(P)=h^{2}\left(\alpha_{i j 8}-\alpha_{i, j+1,8}\right) & \text { of the common side } \\
w(P)=h^{5}\left(\alpha_{i j 6}-\alpha_{i, j+1,6}+4 a \beta\right) & \text { of } K_{i j}, K_{i, j+1} \in \mathcal{C}_{h}^{0} ;\end{cases}
\end{gathered}
$$

(4.13d) the remaining degress of freedom of $z, w, \chi$ and $\zeta$ are equal to zero.

By straightforward computations we find that

$$
\begin{gathered}
(\mu, \chi+\nabla z)=\left(\sum_{k=1}^{3} \mu_{k}, \chi+\nabla z\right) \geqslant C h^{2} \sum_{k=1}^{3}\left\|\mu_{k}\right\|_{0}^{2}, \\
\left(\sum_{k=4}^{8} \mu_{k}, \zeta\right)=\left(\mu_{4}+\mu_{5}+\mu_{8,1}, \zeta\right) \geqslant C h^{4}\left(\sigma_{1}^{2}+\sigma_{2}^{2}\right),
\end{gathered}
$$

and

$$
\left(\sum_{k=4}^{7} \mu_{k}+\mu_{80}, \nabla w\right) \geqslant C h^{6} \sigma_{3}^{2},
$$

where

$$
\begin{aligned}
& \sigma_{1}^{2}=\sum_{i} \sum_{j=1}^{m-1}\left(\alpha_{i j 4}-\alpha_{i, j+1,4}\right)^{2}+\sum_{i=1}^{n-1} \sum_{j}\left(\alpha_{i j 5}-\alpha_{i+1, j 5}\right)^{2}, \\
& \sigma_{2}^{2}=\sum_{i} \sum_{j=1}^{m-1}\left(\alpha_{i j 8}-\alpha_{i, j+1,8}\right)^{2}+\sum_{i=1}^{n-1} \sum_{j}\left(\alpha_{i j 8}-\alpha_{i+1, j 8}\right)^{2},
\end{aligned}
$$


and

$$
\boldsymbol{\sigma}_{3}^{2}=\sum_{i} \sum_{j=1}^{m-1}\left(f_{i j}\right)^{2}+\sum_{i=1}^{n-1} \sum_{j}\left(g_{i j}\right)^{2}
$$

where

$$
\left\{\begin{array}{l}
f_{i j}=\alpha_{i j 6}-\alpha_{i, j+1,6}+4 a \beta \\
g_{i j}=\alpha_{i+1, j 7}-\alpha_{i j 7}-4 b \beta
\end{array}\right.
$$

We shall now estimate the seminorms $\sigma_{i}$ from below using the fact that since $\mu \in N_{h}^{\perp}$ the relations (4.9) and (4.10) hold. First, from (4.9) we conclude that

$$
\sigma_{1}^{2} \geqslant C\left(\left\|\mu_{4}\right\|_{0}^{2}+\left\|\mu_{5}\right\|_{0}^{2}\right)
$$

Next, by (4.12) and Lemma 3.3 with $q=2$, we have

$$
\begin{aligned}
\sigma_{2}^{2} & =\sum_{i} \sum_{j=1}^{m-1}\left(\tilde{\alpha}_{i j 8}-\tilde{\alpha}_{i, j+1,8}\right)^{2}+\sum_{i=1}^{n-1} \sum_{j}\left(\tilde{\alpha}_{i j 8}-\tilde{\alpha}_{i+1, j 8}\right)^{2} \\
& \geqslant C h^{2} \sum_{i, j}\left(\tilde{\alpha}_{i j 8}\right)^{2} \geqslant C_{1}\left\|\mu_{81}\right\|_{0}^{2} .
\end{aligned}
$$

Finally, to estimate $\sigma_{3}$ from below, let us combine (4.15) and (4.9) and solve the resulting system of equations for $\alpha_{i j 6}$ and $\alpha_{i j 7}$ to obtain

$$
\begin{aligned}
& \alpha_{i j 6}=\sum_{l=1}^{m-1} c_{l j}^{m} f_{i l}+2(2 j-m-1) a \beta, \\
& \alpha_{i j 7}=-\sum_{l=1}^{n-1} c_{i l}^{n} g_{l j}+2(2 i-n-1) b \beta, \quad 1 \leqslant i \leqslant n, 1 \leqslant j \leqslant m,
\end{aligned}
$$

where

$$
\begin{aligned}
c_{j l}^{m} & =-l / m, & & \text { if } l \leqslant j-1, \\
& =1-l / m, & & \text { if } l>j-1 .
\end{aligned}
$$

Upon substituting these expressions into (4.10), we obtain a relation of the form

$$
\sum_{i} \sum_{j=1}^{m-1} c_{i j} f_{i j}+\sum_{i=1}^{n-1} \sum_{j} d_{i j} g_{i j}+e \beta=0
$$

where the coefficients $c_{i j}, d_{i j}$ and $e$ satisfy

$$
\left|c_{i j}\right| \leqslant C m^{2}, \quad\left|d_{i j}\right| \leqslant C n^{2}, \quad e \geqslant C\left(n^{3} m+n m^{3}\right) .
$$

Since $n \leqslant C h^{-1}, m \leqslant C h^{-1}$, and $\left\|\mu_{8,0}\right\|_{0}=C \beta$, it follows that

$$
\left\|\mu_{8,0}\right\|_{0} \leqslant \operatorname{Ch}\left\{\sum_{i} \sum_{j=1}^{m-1}\left(f_{i j}\right)^{2}+\sum_{i=1}^{n-1} \sum_{j}\left(g_{i j}\right)^{2}\right\}^{1 / 2} \equiv C h \sigma_{3} .
$$

Moreover, from (4.9) it follows easily that

$$
\sum_{j=1}^{m-1}\left(\alpha_{i j 6}-\alpha_{i, j+1,6}\right)^{2} \geqslant C h^{2} \sum_{j}\left(\alpha_{i j 6}\right)^{2}, \quad 1 \leqslant i \leqslant n,
$$


and

$$
\sum_{i=1}^{n-1}\left(\alpha_{i j 7}-\alpha_{i+1, j 7}\right)^{2} \geqslant C h^{2} \sum_{i}\left(\alpha_{i j 7}\right)^{2}, \quad 1 \leqslant j \leqslant m .
$$

Combining the last three inequalities and recalling the definition of $\sigma_{3}$, we finally get the following lower bound for $\sigma_{3}$ :

$$
\sigma_{3}^{2} \geqslant C\left\{h^{-2}\left\|\mu_{8,0}\right\|_{0}^{2}+h^{2} \sum_{k=6,7} \sum_{i, j}\left(\alpha_{i j k}\right)^{2}\right\} .
$$

Now, let $\delta \in(0,1]$ be a constant to be determined, let $v=-z-\delta^{2} w$ and $\varphi=\chi+\delta \zeta$, and define

$$
\mathcal{H}=\left\{h^{2} \sum_{k=1}^{3}\left\|\mu_{k}\right\|_{0}^{2}+h^{4}\left(\sigma_{1}^{2}+\sigma_{2}^{2}\right)+h^{6} \sigma_{3}^{2}\right\}^{1 / 2} .
$$

Recalling (4.13) and (4.14), we see that

$$
\|\varphi\|_{1}+h^{-1}\|v\|_{1} \leqslant C \mathcal{H C}
$$

and

$$
\begin{aligned}
(\mu, \varphi-\nabla v) \geqslant & C\left\{h^{2} \sum_{k=1}^{3}\left\|\mu_{k}\right\|_{0}^{2}+\delta h^{4}\left(\sigma_{1}^{2}+\sigma_{2}^{2}\right)+\delta^{2} h^{6} \sigma_{3}^{2}\right\} \\
& +\left(\sum_{k=1}^{3} \mu_{k}, \delta \zeta-\delta^{2} \nabla w\right)-\left(\mu_{8,1}, \delta^{2} \nabla w\right) .
\end{aligned}
$$

Using here the easy-to-check estimates

$$
\|\zeta\|_{0} \leqslant C h^{3}\left(\sigma_{1}^{2}+\sigma_{2}^{2}\right)^{1 / 2}, \quad\|\nabla w\|_{0} \leqslant C h^{5} \sigma_{3},
$$

together with (4.17), we find that

$$
(\mu, \varphi-\nabla v) \geqslant\left(C-C_{1} \delta\right)\left\{h^{2} \sum_{k=1}^{3}\left\|\mu_{k}\right\|_{0}^{2}+\delta h^{4}\left(\sigma_{1}^{2}+\sigma_{2}^{2}\right)+\delta^{2} h^{6} \sigma_{3}^{2}\right\} .
$$

Taking now $\delta=\min \left\{1, C / 2 C_{1}\right\}$, we obtain

$$
(\mu, \varphi-\nabla v) \geqslant C \mathcal{H}^{2} \text {. }
$$

Finally, by combining (4.16), (4.17) and (4.19) noting that $\left\|\mu_{8}\right\|_{0}^{2}=\left\|\mu_{80}\right\|_{0}^{2}+$ $\left\|\mu_{81}\right\|_{0}^{2}$, we see that

$$
\mathcal{K} \geqslant C\|\mu\|_{h} .
$$

The desired result now follows from (4.20)-(4.22) and the proof is complete.

In the remaining two lemmas we shall use the following mesh-dependent seminorm:

$$
|\varphi|_{0, h}=h^{-2}\left\{\sum_{\Delta \in \mathcal{C}_{h}} \sum_{i=1}^{2}\left|\int_{\Delta} \varphi_{i} d x d y\right|^{2}\right\}^{1 / 2}, \quad \varphi=\left(\varphi_{1}, \varphi_{2}\right) \in\left[L_{2}(\Omega)\right]^{2} .
$$


LEMMA 4.2. There exists a positive constant $C$ such that for all $(v, \varphi) \in V_{h} \times W_{h}$,

$$
\sup _{\mu \in N_{h}^{\perp}} \frac{(\mu, \varphi-\nabla v)}{h\|\mu\|_{0}} \geqslant C|\varphi-\nabla v|_{0, h} .
$$

Proof. Given $(v, \varphi) \in V_{h} \times W_{h}$, let $\mu \in Q_{h}$ be defined by the relation

$$
(\mu, \zeta)=(\varphi-\nabla v, \zeta) \quad \forall \zeta \in Q_{h}
$$

i.e.,

$$
\left.\mu\right|_{\Delta}=\frac{1}{\operatorname{area}(\Delta)} \int_{\Delta}(\varphi-\nabla v) d x d y \quad \forall \Delta \in \mathcal{C}_{h}
$$

Then $\mu \in N_{h}^{\perp}$ and

$$
(\mu, \varphi-\nabla v)=\|\mu\|_{0}^{2}=h^{4}\left(h_{1} h_{2}\right)^{-1}|\varphi-\nabla v|_{0, h}^{2} \geqslant C h^{2}|\varphi-\nabla v|_{0, h}^{2},
$$

which proves the desired result.

LEMMA 4.3. There is a constant $C$ such that for $(v, \varphi) \in V_{h} \times W_{h}$

$$
|v|_{1} \leqslant C\left(|\varphi-\nabla v|_{0, h}+|\varphi|_{1}\right) \text {. }
$$

Proof. Let $(v, \varphi)=\left(v, \varphi_{1}, \varphi_{2}\right) \in V_{h} \times W_{h}$ be given. We denote by $\left(x_{i}, y_{j}\right)=$ $(i-1) h_{1} / 2,(j-1) h_{2} / 2$ the nodes of $\mathcal{C}_{h}$ so that if $\Delta_{i j} \in \mathcal{C}_{h}$, then $\Delta_{i j}=$ $\left\{(x, y) ; x_{i}<x<x_{i+1}, y_{j}<y<y_{j+1}\right\}$. Using the notation

$$
w_{i, j}=w\left(x_{i}, y_{j}\right), \quad w_{i+1 / 2, j+1 / 2}=w\left(x_{i}+\frac{h_{1}}{4}, y_{j}+\frac{h_{2}}{4}\right),
$$

we have if $\left(x_{i}, y_{j}\right)$ is an interior node

$$
\begin{aligned}
& \left(\varphi_{1}-\frac{\partial v}{\partial x}\right)_{i+1 / 2, j+1 / 2}=\frac{1}{h_{1}}\left(v_{i j}-v_{i+1, j}+v_{i, j+1}-v_{i+1, j+1}\right)+\varphi_{1, i+1 / 2, j+1 / 2}, \\
& \left(\varphi_{2}-\frac{\partial v}{\partial y}\right)_{i+1 / 2, j+1 / 2}=\frac{1}{h_{2}}\left(v_{i j}-v_{i, j+1}+v_{i+1, j}-v_{i+1, j+1}\right)+\varphi_{2, i+1 / 2, j+1 / 2} .
\end{aligned}
$$

Adding these equations we find that

$$
v_{i+1, j+1}-v_{i j}=f_{i+1 / 2, j+1 / 2},
$$

where

$$
f=\frac{1}{2}\left[h_{1} \varphi_{1}+h_{2} \varphi_{2}-h_{1}\left(\varphi_{1}-\frac{\partial v}{\partial x}\right)-h_{2}\left(\varphi_{2}-\frac{\partial v}{\partial y}\right)\right] .
$$

Since $v$ vanishes on the boundary of $\Omega$, we have $v_{i-j+1,1}=0$ if $i \geqslant j$ and thus (4.23) may be solved for $v_{i j}$ to obtain

$$
v_{i j}=\sum_{\nu=1}^{j-1} f_{i-\nu+1 / 2, j-\nu+1 / 2}
$$

Therefore, since $j \leqslant C h^{-1}$,

$$
\begin{aligned}
\left(v_{i+1, j}-v_{i j}\right)^{2} & =\left[\sum_{\nu=1}^{j-1}\left(f_{i-\nu+3 / 2, j-\nu+1 / 2}-f_{i-\nu+1 / 2, j-\nu+1 / 2}\right)\right]^{2} \\
& \leqslant C h^{-1} \sum_{\nu=1}^{j-1}\left(f_{i-\nu+3 / 2, j-\nu+1 / 2}-f_{i-\nu+1 / 2, j-\nu+1 / 2}\right)^{2}
\end{aligned}
$$


An analogous estimate can be derived for $\left(v_{i, j+1}-v_{i j}\right)^{2}$, and, combining these estimates and summing over $i$ and $j$, we see that

$$
\begin{aligned}
|v|_{1}^{2} & \leqslant C \sum_{i, j}\left\{\left(v_{i+1, j}-v_{i j}\right)^{2}+\left(v_{i, j+1}-v_{i j}\right)^{2}\right\} \\
& \leqslant C_{1} h^{-2} \sum_{i, j}\left\{\left(f_{i+3 / 2, j+1 / 2}-f_{i+1 / 2, j+1 / 2}\right)^{2}+\left(f_{i+1 / 2, j+3 / 2}-f_{i+1 / 2, j+1 / 2}\right)^{2}\right\} .
\end{aligned}
$$

Further, by the definition of $f$ we have

$$
\begin{aligned}
\left(f_{i+3 / 2, j+1 / 2}-\right. & \left.f_{i+1 / 2, j+1 / 2}\right)^{2} \\
\leqslant & C h^{2} \sum_{k=i}^{i+1} \sum_{l=j}^{j+1}\left\{\left(\varphi_{1, k+1, l}-\varphi_{1 k l}\right)^{2}+\left(\varphi_{2, k+1, l}-\varphi_{2 k l}\right)^{2}\right\} \\
& +C h^{2} \sum_{k=i}^{i+1}\left\{\left(\varphi_{1}-\frac{\partial v}{\partial x}\right)_{k+1 / 2, j+1 / 2}^{2}+\left(\varphi_{2}-\frac{\partial v}{\partial y}\right)_{k+1 / 2, j+1 / 2}^{2}\right\}
\end{aligned}
$$

Together with a similar inequality for $\left(f_{i+1 / 2, j+3 / 2}-f_{i+1 / 2, j+1 / 2}\right)^{2}$ this shows that

$$
\begin{aligned}
|v|_{1}^{2} \leqslant & C \sum_{i, j} \sum_{k=1}^{2}\left\{\left(\varphi_{k, i+1, j}-\varphi_{k, i j}\right)^{2}+\left(\varphi_{k i, j+1}-\varphi_{k i j}\right)^{2}\right\} \\
& +C \sum_{i, j}\left\{\left(\varphi_{1}-\frac{\partial v}{\partial x}\right)_{i+1 / 2, j+1 / 2}^{2}+\left(\varphi_{2}-\frac{\partial v}{\partial y}\right)_{i+1 / 2, j+1 / 2}^{2}\right\} \\
\leqslant & C_{1}\left(|\varphi|_{1}^{2}+|\varphi-\nabla v|_{0, h}^{2}\right),
\end{aligned}
$$

and the desired estimate follows.

We can now state and prove the main result of this section.

THEOREM 4.1. There is a constant $C$ such that if $u \in H^{5}(\Omega)$ satisfies (4.1) and $\left(u_{h}, \lambda_{h}, \theta_{h}\right)$ is the solution of the discrete problem (4.7), with $0<\varepsilon \leqslant C h^{2}$, then

$$
\left\|u-u_{h}\right\|_{1}+\left\|\nabla u-\theta_{h}\right\|_{1} \leqslant C h\|u\|_{5} .
$$

Proof. Let $(\tilde{u}, \tilde{\theta}) \in V_{h} \times W_{h}$ be the usual interpolant of $(u, \theta)$, and let $\tilde{\lambda}$ be the orthogonal projection of $\lambda$ onto $N_{h}^{\perp}$. By (4.7) and (4.8) (with $\varepsilon=0$ in (4.8)), we have

$$
\begin{aligned}
& \mathscr{B}\left(u_{h}-\tilde{u}, \theta_{h}-\tilde{\theta}, \lambda_{h}-\tilde{\lambda} ; v, \varphi, \mu\right) \\
&=\mathscr{B}(u-\tilde{u}, \boldsymbol{\theta}-\tilde{\theta}, \lambda-\tilde{\lambda} ; v, \varphi, \mu)-\varepsilon(\lambda, \mu) \equiv R H, \\
& \forall(v, \varphi, \mu) \in V_{h} \times W_{h} \times Q_{h},
\end{aligned}
$$

where $\theta=\nabla u, \lambda=\Delta \theta$ and

$$
\Re(u, \theta, \lambda ; v, \varphi, \mu)=(\nabla \theta, \nabla \varphi)-(\mu, \theta-\nabla u)+(\lambda, \varphi-\nabla v)+\varepsilon(\lambda, \mu) .
$$

Since by (4.7c) $\lambda_{h} \in N_{h}^{\perp}$, we have $\lambda_{h}-\tilde{\lambda} \in N_{h}^{\perp}$ and thus, by Lemma 4.1 , there exists $(z, \zeta) \in V_{h} \times W_{h}$ such that

$$
\begin{gathered}
\|z\|_{1}+\|\zeta\|_{1} \leqslant C\left\|\lambda_{h}-\tilde{\lambda}\right\|_{h}, \\
\left(\zeta-\nabla z, \lambda_{h}-\tilde{\lambda}\right) \geqslant\left\|\lambda_{h}-\tilde{\lambda}\right\|_{h}^{2} .
\end{gathered}
$$

Further, by Lemma 4.2 and (4.11), there exists $\nu \in N_{h}^{\perp}$ such that

$$
\|\nu\|_{h} \leqslant C h\|\nu\|_{0} \leqslant C_{1}\left|\theta_{h}-\tilde{\theta}-\nabla\left(u_{h}-\tilde{u}\right)\right|_{0, h},
$$




$$
-\left(\nu, \theta_{h}-\tilde{\theta}-\nabla\left(u_{h}-\tilde{u}\right)\right) \geqslant\left|\theta_{h}-\tilde{\theta}-\nabla\left(u_{h}-\tilde{u}\right)\right|_{0, h}^{2} .
$$

Let now $v=u_{h}-\tilde{u}+\delta z, \varphi=\theta_{h}-\tilde{\theta}+\delta \zeta$ and $\mu=\lambda_{h}-\tilde{\lambda}+\delta \nu$, where $\delta \in(0,1]$ will be chosen below. Then, by Lemma 4.3 and the fact that $\varepsilon \leqslant C h^{2}$, we have

$$
\|v\|_{1}+\|\varphi\|_{1}+\|\nu\|_{h}+\sqrt{\varepsilon}\|\mu\|_{0} \leqslant C \mathcal{H},
$$

where

$$
\begin{aligned}
\mathcal{C}=\left\{\left|\theta_{h}-\tilde{\theta}\right|_{1}^{2}+\left|\theta_{h}-\tilde{\theta}-\nabla\left(u_{h}-\tilde{u}\right)\right|_{0, h}^{2}\right. \\
\\
\left.+\left\|\lambda_{h}-\tilde{\lambda}\right\|_{h}^{2}+\varepsilon\left\|\lambda_{h}-\tilde{\lambda}\right\|_{0}^{2}\right\}^{1 / 2} .
\end{aligned}
$$

Further, by (4.25)-(4.28) we have

$$
\begin{aligned}
\mathfrak{B}\left(u_{h}-\tilde{u}, \theta_{h}-\tilde{\theta}, \lambda_{h}-\tilde{\lambda} ; v, \varphi, \mu\right) & \\
\geqslant & \left|\theta_{h}-\tilde{\theta}\right|_{1}^{2}+\delta\left|\theta_{h}-\tilde{\theta}-\nabla\left(u_{h}-\tilde{u}\right)\right|_{0, h}^{2}+\delta\left\|\lambda_{h}-\tilde{\lambda}\right\|_{h}^{2} \\
& +\varepsilon\left\|\lambda_{h}-\tilde{\lambda}\right\|_{0}^{2}+\left(\nabla\left(\theta_{h}-\tilde{\theta}\right), \delta \nabla \zeta\right)+\varepsilon\left(\lambda_{h}-\tilde{\lambda}, \delta \nu\right) \\
\geqslant & (1-C \delta)\left|\theta_{h}-\tilde{\theta}\right|_{1}^{2}+\delta\left(1-C \delta \varepsilon h^{-2}\right)\left|\theta_{h}-\tilde{\theta}-\nabla\left(u_{h}-\tilde{u}\right)\right|_{0, h}^{2} \\
& \quad+\frac{1}{2} \delta\left\|\lambda_{h}-\tilde{\lambda}\right\|_{h}^{2}+\frac{1}{2} \varepsilon\left\|\lambda_{h}-\tilde{\lambda}\right\|_{0}^{2} .
\end{aligned}
$$

Choosing here $\delta=\min \left\{1,1 / 2 C, h^{2} / 2 C \varepsilon\right\}$, we obtain

$$
\mathscr{B}\left(u_{h}-\tilde{u}, \theta_{h}-\tilde{\theta}, \lambda_{h}-\tilde{\lambda} ; v, \varphi, \mu\right) \geqslant C \mathcal{F}^{2} .
$$

Next, to estimate the right-hand side $R H$ of (4.24) we first note that, by standard interpolation theory and (4.29),

$$
|(\nabla(\theta-\tilde{\theta}), \nabla \varphi)| \leqslant C \mathcal{F C} h|\theta|_{2} \leqslant C \mathcal{H C h}|u|_{3},
$$

and also by (4.29)

$$
|\varepsilon(\tilde{\lambda}, \mu)| \leqslant C \mathcal{F} \sqrt{\varepsilon}\|\lambda\|_{0} \leqslant C_{1} \mathcal{F C h}|u|_{3} .
$$

Further, denoting by $\pi_{h} \lambda$ the orthogonal projection of $\lambda$ onto $Q_{h}$, we have by (4.29)

$$
\begin{aligned}
|(\lambda-\tilde{\lambda}, \varphi-\nabla v)| & =\left|\left(\lambda-\pi_{h} \lambda, \varphi-\nabla v\right)\right| \\
& \leqslant C \mathcal{H C}\left\|\lambda-\pi_{h} \lambda\right\|_{0} \leqslant C_{1} \mathcal{H C} h|\lambda|_{1} \leqslant C_{1} \mathcal{S C} h|u|_{4} .
\end{aligned}
$$

To estimate $|(\mu, \theta-\tilde{\theta}-\nabla(u-\tilde{u}))|$, we first note that, by the definition of the seminorm $\|\cdot\|_{h}$ and the fact that $\|\mu\|_{h} \leqslant C \mathcal{H}$, we have

$$
|(\mu, \theta-\tilde{\theta}-\nabla(u-\tilde{u}))| \leqslant C \mathcal{H} \sum_{k=1}^{3} \Gamma_{k}(\theta-\tilde{\theta}-\nabla(u-\tilde{u})),
$$

where

$$
\begin{aligned}
\Gamma_{1}(\chi) & =h^{-2}\left[\sum_{k=1}^{3} \gamma_{k}(\chi)^{2}\right]^{1 / 2}, \\
\Gamma_{2}(\chi) & =h^{-3}\left[\sum_{k=4,5,8} \gamma_{k}(\chi)^{2}\right]^{1 / 2}, \\
\Gamma_{3}(\chi) & =h^{-4}\left[\sum_{k=6,7} \gamma_{k}(\chi)^{2}\right]^{1 / 2}, \\
\gamma_{k}(\chi)^{2} & =\sum_{i j} \gamma_{i j k}(\chi)^{2}, \quad \gamma_{i j k}(\chi)=\left|\int_{K_{i j}} \chi \eta_{i j k} d x d y\right| .
\end{aligned}
$$


Now, recalling Lemma 3.4, we easily find that

$$
\begin{aligned}
\Gamma_{1}(\theta-\tilde{\theta}-\nabla(u-\tilde{u})) & \leqslant C^{-2}\left\{\sum_{\Delta \in \bigodot_{h}}\left|\int_{\Delta}(\theta-\tilde{\theta}-\nabla(u-\tilde{u})) d x d y\right|^{2}\right\}^{1 / 2} \\
& \leqslant C h\left(|\theta|_{2}+|u|_{3}\right) .
\end{aligned}
$$

To estimate $\Gamma_{2}$ and $\Gamma_{3}$ we shall use the following additional superapproximation result, the proof of which is straightforward.

Lemma 4.4. Defining for $(v, \varphi) \in H^{1}(Q) \times\left[H^{1}(Q)\right]^{2}, Q=K_{i j} \in \bigodot_{h}^{0}$,

$$
L_{k}(v, \varphi)=\int_{Q}(\varphi-\tilde{\varphi}-\nabla(v-\tilde{v})) \eta_{i j k} d x d y
$$

where ${ }^{\sim}$ denotes the piecewise bilinear interpolant on the four subrectangles of $K_{i j}$, we have

$$
\begin{array}{ll}
L_{k}(v, \varphi)=0 & \text { if }(v, \varphi) \in P_{3} \times\left[P_{2}\right]^{2} \text { for } k=4,5,8 \\
L_{k}(v, \varphi)=0 & \text { if }(v, \varphi) \in P_{4} \times\left[P_{3}\right]^{2} \text { for } k=6,7
\end{array}
$$

so that

$$
\begin{array}{ll}
\left|L_{k}(v, \varphi)\right| \leqslant C h^{4}\left(|\theta|_{H^{3}(Q)}+|u|_{H^{4}(Q)}\right) & \text { for } k=4,5,8 \\
\left|L_{k}(v, \varphi)\right| \leqslant C h^{5}\left(|\theta|_{H^{4}(Q)}+|u|_{H^{5}(Q)}\right) & \text { for } k=6,7 .
\end{array}
$$

From this lemma we conclude that

$$
\begin{aligned}
& \Gamma_{2}(\theta-\tilde{\theta}-\nabla(u-\tilde{u})) \leqslant C h\left(|\theta|_{3}+|u|_{4}\right), \\
& \Gamma_{3}(\theta-\tilde{\theta}-\nabla(u-\tilde{u})) \leqslant C h\left(|\theta|_{4}+|u|_{5}\right) .
\end{aligned}
$$

Recalling (4.32)-(4.35) and collecting the estimates for $\Gamma_{i}$, we obtain $R H \leqslant$ $C \mathcal{F} h\|u\|_{5}$, which combined with (4.24) and (4.31) shows that $\mathcal{H} \leqslant C h\|u\|_{5}$. Together with the usual estimates for the interpolation error this proves the stated estimates for $\left\|u-u_{h}\right\|_{1}$ and $\left\|\boldsymbol{\theta}-\boldsymbol{\theta}_{\boldsymbol{h}}\right\|_{1}$, and the proof is complete.

Remark. In general the solution $u$ of (4.1) does not belong to $H^{5}(\Omega)$. The best one can say in general is that $u \in H^{s}(\Omega)$ with $s \sim 4.73$ if $f \in H^{1}(\Omega)$ (cf. [12]). Replacing $\|\cdot\|_{h}$ by a slightly stronger norm, which is possible since in the proof of Lemma 4.1 Sobolev imbedding was used, one can prove that the statement of Theorem 4.1 holds with $\|u\|_{5}$ replaced by $\|f\|_{1}$.

Remark. Due to the extra smoothness required to use the superapproximability property, the usual duality argument does not give the optimal rate $\theta\left(h^{2}\right)$ for $\left\|u-u_{h}\right\|_{0}$. It is possible to prove that $\left\|u-u_{h}\right\|_{0} \leqslant C h^{3 / 2}\|f\|_{1}$.

Department of Mathematics

Chalmers University of Technology

S-41296 Göteborg, Sweden 
1. I. BABUSKK, "Error bounds for finite element methods," Numer. Math., v. 16, 1971, pp. 322-333.

2. I. BabuSKa, J. Osborn \& J. PitkARanta, Analysis of Mixed Methods Using Mesh Dependent Norms, Report \# 2003, Mathematics Research Center, University of Wisconsin, 1979.

3. M. Bercovier, "Perturbation of mixed variational problems. Application to mixed finite element methods," R.A.I.R.O. Anal. Numer., v. 12, 1978, pp. 211-236.

4. F. BREZZI, "On the existence, uniqueness and approximation of saddle-point problems arising from Lagrange multipliers," R.A.I.R.O. Sér. Rouge, v. 8, 1974, pp. 129-151.

5. P. Ciarlet, The Finite Element Method for Elliptic Problems, North-Holland, Amsterdam, 1978.

6. M. Crouzeix \& P.-A. Raviart, "Conforming and nonconforming finite element methods for solving the stationary Stokes equations. I," R.A.I.R.O. Sér. Rouge, v. 7, 1973, pp. 33-76.

7. V. Girault, "A combined finite element and Marker-and-Cell method for solving Navier-Stokes equations," Numer. Math., v. 26, 1976, pp. 39-59.

8. V. Girault \& P.-A. Raviart, Finite Element Approximation of the Navier-Stokes Equations, Lecture Notes in Math., Vol. 749, Springer-Verlag, Berlin, 1979.

9. R. Glowinski \& O. Pironneau, "On numerical methods for the Stokes problem," in Energy Methods in Finite Element Analysis (Glowinski et al., Eds.), Wiley, New York, 1979.

10. V. KondRAT'Ev, "Boundary problems for elliptic equations in domains with conical or angular points," Trans. Moscow Math. Soc., v. 16, 1967, pp. 227-313.

11. D. Malkus \& T. Hughes, "Mixed finite element methods-Reduced and selective integration techniques: A unification of concepts," Comput. Methods Appl. Mech. Engrg., v. 15, 1978, pp. 63-81.

12. H. Melzer \& R. RANNACHER, Spannungskonzentrationen in Eckpunkten der vertikalen belasteten Kirchoffschen Platte, Universität Bonn, 1979. (Preprint.)

13. R. L. SANI, P. M. Gresho \& R. L. Lee, On the Spurious Pressures Generated by Certain GFEM Solutions of the Incompressible Navier-Stokes Equations, Technical report, Lawrence Livermore Laboratory, Oct. 1979.

14. K. SiNGH, "Reduced integration for improved accuracy of finite element approximations," Comput. Methods Appl. Mech. Engrg., v. 14, 1978, pp. 23-37.

15. R. Temam, "Une méthode d'approximation de la solution des equations Navier-Stokes," Bull. Soc. Math. France, v. 98, 1968, pp. 115-152. 